\title{
On Nonnegative Solutions of Fractional $q$-Linear Time-Varying Dynamic Systems with Delayed Dynamics
}

\author{
M. De la Sen \\ Institute for Research and Development of Processes, Faculty of Science and Technology, University of Basque Country, \\ Campus of Leioa, Barrio Sarriena, P.O. Box 48940, Leioa, Spain
}

Correspondence should be addressed to M. De la Sen; manuel.delasen@ehu.es

Received 8 January 2014; Accepted 8 February 2014; Published 8 May 2014

Academic Editor: Dumitru Baleanu

Copyright (C) 2014 M. De la Sen. This is an open access article distributed under the Creative Commons Attribution License, which permits unrestricted use, distribution, and reproduction in any medium, provided the original work is properly cited.

This paper is devoted to the investigation of nonnegative solutions and the stability and asymptotic properties of the solutions of fractional differential dynamic linear time-varying systems involving delayed dynamics with delays. The dynamic systems are described based on $q$-calculus and Caputo fractional derivatives on any order.

\section{Introduction}

There are abundant background results concerning the exact and approximate solutions of fractional differential equations [1-4], fractional derivatives involving products of polynomials $[5,6]$, fractional derivatives and fractional powers of operators [7-9], and boundary value problems concerning fractional calculus in a theoretical context and also concerning a wide range of applications like, for instance, control theory, robotics, signal processing, heat transfer, lossless transmission lines, and so forth [1-22]. In particular, some generalized operators of fractional integration have been recently applied to the product of generalized Bessel functions of the first class in [6] leading to multivariable generalized Lauricella-type functions. Furthermore, related generalized fractional integrals are also discussed in that paper. On the other hand, new unified integral formulas involving products of Srivastava-type polynomials and the $H$-function as well as fractional integration for Appell's functions are discussed in $[23,24]$.

This paper is concerned with the investigation of nonnegative solutions of fractional $q$-differential dynamic systems with point delays and some related asymptotic properties formulated by the Caputo fractional derivative. See, for instance, [25-31] for formulations of functional dynamic systems under delays. Some of these papers are concerned with fundamental properties of positive dynamic systems or with the nonnegative solutions of dynamic systems in a fractional context. See, for instance, [32-34]. On the other hand, see [13-22, 32-39] for a background on quantum and fractional calculus and some related applications to dynamic systems.

The Caputo q-difference scheme has been proposed in [40] and then the problems of initial values are investigated in $[41,42]$. In particular, a Caputo-type $q$-fractional initial value problem is solved in [41] with the solution being formulated in terms of a new q-Mittag-Leffler function. On the other hand, the related investigation in [42] is focused on analytical aspects of $q$-fractional calculus while the variational iteration method is extended "ad hoc" to $q$-fractional calculus in order to solve the Caputo $q$-fractional initial value problem. There is also a recent monograph [43] available on the fractional $q$-difference methodology which is of potential interest for readers interested in quantum fractional calculus. Also, it has to be pointed out that an increasing research interest is being devoted to the use of fractional calculus in the analysis of mathematical models based on partial differential equations. In particular, the fractal heat conduction problem is solved by proposing a local fractional variation iteration method in [44]. On the other hand, the solutions of the Helmholtz equation involving local fractional derivative operators are investigated in [45] combined with series expansion and variational iteration methods. 
It might be pointed out that positive dynamic models are an essential tool to describe some real world applications as, for instance, medical, biological, or epidemic models. It has to be pointed out that a major advantage of the use of $q$ calculus is that it does not need the existence of limits or restrictive regularity conditions on the functions dealt with in order to establish the formulation. In particular, derivatives and higher-order derivatives of a wide class of functions exist almost everywhere under the $q$-calculus framework [37]. In this context, the $q$-calculus formalism on differ-integral systems is close to the classical one on difference systems with the additional advantage that the parameter running the samples can be chosen to be real so that it links the selection of the sampling points in a multiplicative fashion. Such sampling points are backward-in-time dependent on each time instant for which the $q$-fractional solution is evaluated while asymptotically vanishing to zero as the number of used samples increases to infinity for each given time instant where the quantum fractional solution is being computed.

1.1. Notation. $\mathbf{Z}, \mathbf{R}$, and $\mathbf{C}$ are the sets of integer, real, and complex numbers, $\mathbf{Z}_{+}$and $\mathbf{R}_{+}$are the positive integer and real numbers, and

$$
\begin{gathered}
\mathbf{N} \equiv \mathbf{Z}_{0+}:=\mathbf{Z}_{+} \cup\{0\} ; \quad \mathbf{R}_{0+}:=\mathbf{R}_{+} \cup\{0\} ; \\
\mathbf{C}_{+}:=\{z \in \mathbf{C}: \operatorname{Re} z>0\} ; \quad \mathbf{C}_{0+}:=\{z \in \mathbf{C}: \operatorname{Re} z \geq 0\}, \\
\bar{n}:=\{1,2, \ldots, n\} .
\end{gathered}
$$

The following notation is used to characterize different levels of positivity of matrices.

$\mathbf{R}_{0+}^{n \times m}:=\left\{M=\left(m_{i j}\right) \in \mathbf{R}^{n \times m}: m_{i j} \geq 0\right.$; for all $(i, j) \epsilon$ $\bar{n} \times \bar{m}\}$ is the set of all $n \times m$ real matrices of nonnegative entries. If $M \in \mathbf{R}^{n \times m}$ then $M \geq 0$ is used as a simpler notation for $M \in \mathbf{R}_{0+}^{n \times m}$.

$\mathbf{R}_{+}^{n \times m}:=\left\{0 \neq M=\left(m_{i j}\right) \in \mathbf{R}^{n \times m}: m_{i j} \geq 0\right.$; for all $(i, j) \in$ $\bar{n} \times \bar{m}\}$ is the set of all nonzero $n \times m$ real matrices of nonnegative entries (i.e., those with at least one of their entries being positive). If $M \in \mathbf{R}^{n \times m}$ then $M>0$ is used as a simpler notation for $M \in \mathbf{R}_{+}^{n \times m}$.

$\mathbf{R}_{++}^{n \times m}:=\left\{M=\left(M_{i j}\right) \in \mathbf{R}^{n \times m}: M_{i j}>0\right.$; for all $(i, j) \epsilon$ $\bar{n} \times \bar{m}\}$ is the set of all $n \times m$ real matrices of positive entries. If $M \in \mathbf{R}^{n \times m}$ then $M \gg 0$ is used as a simpler notation for $M \in \mathbf{R}_{++}^{n \times m}$. The superscript $T$ denotes the transpose and $M_{i}^{T}$ and $M_{j}$ are, respectively, the $i$ th row and the $j$ th column of the matrix $M$.

A close notation to characterize the positivity of vectors is the following.

$\mathbf{R}_{0+}^{n}:=\left\{v=\left(v_{1}, v_{2}, \ldots, v_{n}\right)^{T} \in \mathbf{R}^{n}: v_{i} \geq 0\right.$; for all $\left.i \in \bar{n}\right\}$ is the set of all $n$ real vectors of nonnegative components. If $v \in \mathbf{R}^{n}$ then $v \geq 0$ is used as a simpler notation for $v \in \mathbf{R}_{0+}^{n}$.

$\mathbf{R}_{+}^{n}:=\left\{0 \neq v=\left(v_{1}, v_{2}, \ldots, v_{n}\right)^{T} \in \mathbf{R}^{n}: v_{i} \geq 0\right.$; for all $i \in$ $\bar{n}\}$ is the set of all $n$ real nonzero vectors of nonnegative components (i.e., at least one component is positive). If $v \in \mathbf{R}^{n}$ then $v>0$ is used as a simpler notation for $v \in \mathbf{R}_{+}^{n}$.

$\mathbf{R}_{++}^{n}:=\left\{v=\left(v_{1}, v_{2}, \ldots, v_{n}\right)^{T} \in \mathbf{R}^{n}: v_{i}>0\right.$; for all $\left.i \in \bar{n}\right\}$ is the set of all $n$ real vectors of positive components. If $v \in \mathbf{R}^{n}$ then $v \gg 0$ is used as a simpler notation for $v \in \mathbf{R}_{++}^{n}$.
$M=\left(M_{i j}\right) \in \mathbf{R}^{n \times n}$ is a Metzler matrix if $M_{i j} \geq$ 0 ; for all $(i, j \neq i) \in \bar{n} \times \bar{n}$. $M \mathbf{R}^{n \times n}$ is the set of Metzler matrices of order $n$.

The maximum real eigenvalue, if any, of a real matrix $M$, is denoted by $\lambda_{\max }(M)$.

$x \geq y, x>y$, and $x \gg y$ mean, respectively, $x-y \geq 0$, $x-y>0$, and $x-y \gg 0$ for $x, y$ being any real scalars, vectors, or matrices of compatible dimensions or orders.

The following fundamental result of [32] is concerned with the unique left-sided solution on $\mathbf{R}_{0+}$ of the differential fractional system (36).

Theorem 1. Consider the Caputo fractional differential system of order $\alpha$ with $p \geq 0$ (potentially repeated) delays and $0 \leq q \leq$ $p$ distinct delays:

$$
\begin{aligned}
& \left({ }^{C} D_{a+}^{\alpha} x\right)(t) \\
& \quad:=\frac{1}{\Gamma(k-\alpha)} \int_{a}^{t}(t-\tau)^{k-\alpha-1}\left(x^{(k)}(\tau)\right) d \tau \\
& \quad=\sum_{i=0}^{p} A_{i}\left(t-h_{i}\right)+B u(t) ; \quad t \in[a, b]
\end{aligned}
$$

on $\mathbf{R}_{0+}$, for any $\alpha \in \mathbf{C}_{0+}$ with $k=[\operatorname{Re} \alpha]+1$ if $\alpha \notin \mathbf{Z}_{+}$ and $k=\alpha$ if $\alpha \in \mathbf{Z}_{+} ; k-1, k \in \mathbf{Z}_{0+}, 0=h_{0}<h_{1}<$ $h_{2}<\cdots<h_{p}=h<\infty$ are distinct constant delays, $A_{0}, A_{i} \in \mathbf{R}^{n \times n}(i \in \bar{p}:=\{1,2, \ldots, p\})$, are the matrices of dynamics for each delay $h_{i}, i \in \bar{p} \cup\{0\}, B \in \mathbf{R}^{n \times m}$ is the control matrix and with initial condition of the state being given by $k n$ real vector functions $\varphi_{j}:[-h, 0] \rightarrow \mathbf{R}^{n}$, with $j \in \overline{k-1} \cup\{0\}$, which are absolutely continuous except eventually in a set of zero measure of $[-h, 0] \subset \mathbf{R}$ of bounded discontinuities with $\varphi_{j}(0)=x_{j}(0)=x^{(j)}(0)=x_{j 0} j \in \overline{k-1} \cup\{0\}$ and $u: \mathbf{R}_{0+} \rightarrow$ $\mathbf{R}^{m}$ is a bounded piecewise continuous control function. Then, the unique left-sided solution of (2) is given by

$$
\begin{aligned}
x(t)= & \sum_{j=0}^{k-1}\left(\Phi_{\alpha j 0}(\tau) x_{j 0}\right. \\
& \left.\quad+\sum_{i=1}^{p} \int_{0}^{h_{i}} \Phi_{\alpha}(t-\tau) A_{i} \varphi_{j}\left(\tau-h_{i}\right) d \tau\right) \\
& +\sum_{i=1}^{p} \int_{h_{i}}^{t} \Phi_{\alpha}(t-\tau) A_{i} x\left(\tau-h_{i}\right) d \tau \\
& +\int_{0}^{t} \Phi_{\alpha}(t-\tau) B u(\tau) d \tau ; \quad t \in \mathbf{R}_{0+}
\end{aligned}
$$

with $k=[\operatorname{Re} \alpha]+1$ if $\alpha \notin \mathbf{Z}_{+}$and $k=\alpha$ if $\alpha \in \mathbf{Z}_{+}$, where

$$
\begin{gathered}
\Phi_{\alpha j 0}(t):=t^{j} E_{\alpha, j+1}\left(A_{0} t^{\alpha}\right) ; \\
\Phi_{\alpha}(t):=t^{\alpha-1} E_{\alpha, \alpha}\left(A_{0} t^{\alpha}\right), \\
E_{\alpha, j}\left(A_{0} t^{\alpha}\right):=\sum_{\ell=0}^{\infty} \frac{\left(A_{0} t^{\alpha}\right)^{\ell}}{\Gamma(\alpha \ell+j)} ; \quad j \in \overline{k-1} \cup\{0, \alpha\}
\end{gathered}
$$


for $t \geq 0$ and $\Phi_{\alpha 0}(t)=\Phi_{\alpha}(t)=0$ for $t<0$, where $E_{\alpha, j}\left(A_{0} t^{\alpha}\right)$ are the Mittag-Leffler functions.

If $q$-calculus is used then the integral formulas of Theorem 1 are not necessary to calculate the fractional Caputo-type solutions of any order as shown in the subsequent example.

Example 2. Consider the differential equation $\dot{x}(t)=a x(t)$, $x(0)=x_{0}$. The standard solution for any real $q \in(0,1)$ and $t \geq 0$ is $x(t)=e^{a(1-q) t} x(q t)$. It is globally stable (resp., globally asymptotically stable) for $a \leq 0$ (resp., for $a<0$ ) and nonnegative for any $a \in \mathbf{R}$ if $x(0) \geq 0$.

The use of $q$-calculus for $D_{q} x(t):=(x(t)-x(q t)) /(1-q) t=$ ax $(t)$ yields

$$
x(t)=x(q t)+(1-q) a t x(t)
$$

then, the solution becomes

$$
x(t)=\frac{x(q t)}{1-(1-q) a t} .
$$

(a) If $a>0$ then it is positive if $x(0) \geq 0$ and $0 \leq$ $t<1 /[(1-q) a]$ but any $q$-solution is unbounded if $x(0) \neq 0$ and $|x(t) / x(q t)|>1$ if $0 \leq t<1 /[(1-q) a]$ and $|x(t) / x(q t)|=+\infty$ if $t=1 /[(1-q) a]$.

(b) If $a=0$ then $x(t)=x(0)$ so that the $q$-solution is constant, globally stable, and nonnegative if $x(0) \geq 0$.

(c) If $a<0$ then $x(t)=x(q t) /(1+(1-q)|a| t) \leq x(q t) \leq$ $x(0) ; t \geq 0$ with the first inequality being obvious and the second one being proved by contradiction. Assume that the second inequality is false. Then, there is $t>0$, so that there is $q t>0$, such that for any $n \in \mathbf{N}$, the subsequent contradiction is got:

$$
\begin{aligned}
x(0) & <x(q t) \\
& =(1+(1-q)|a| t) x(t) \leq x\left(q^{2} t\right) \\
& =(1+(1-q)|a| q t) x(q t) \\
& \leq \cdots \leq\left(1+(1-q)|a| q^{n} t\right) x\left(q^{n+1} t\right) \\
& \leq \limsup _{n \rightarrow+\infty}\left(1+(1-q)|a| q^{n} t\right) x\left(q^{n+1} t\right) \\
& =\lim _{n \rightarrow+\infty}\left(1+(1-q)|a| q^{n} t\right) x\left(q^{n+1} t\right)=x(0) .
\end{aligned}
$$

Since $x(t)$ is bounded for $t \geq 0$ for any given $x(0)$ then $\lim _{t \rightarrow \infty} x(t)=0$. Therefore, for $a<0$ the $q$-differential equation has a nonnegative $q$-solution on $\mathbf{R}_{0+}$ for any $x(0) \geq 0$ and it is globally asymptotically stable. On the other hand, the Caputo fractional solution of real order $\alpha$ of the associate fractional differential equation is

$$
x^{(\alpha)}(t)=\frac{d x^{(\alpha)}(t)}{d t^{\alpha}}=a x(t), \quad x(0)=x_{0}, t \geq 0 .
$$

The formulation within the $q$-calculus framework leads to

$$
\begin{aligned}
& x^{(\alpha-1)}(t)=x^{(\alpha-1)}(0)+\int_{0}^{t} a x\left(\tau_{1}\right) d \tau_{1}, \\
& x^{(\alpha-2)}(t)=x^{(\alpha-2)}(0)+t x^{(\alpha-1)}(0) \\
& +\int_{0}^{t} \int_{0}^{\tau_{1}} a x\left(\tau_{2}\right) d \tau_{2} d \tau_{1} \\
& x^{(\alpha-j)}(t)=\sum_{i=1}^{j} t^{j-i} x^{(j-i)}(0) \\
& +a \int_{0}^{t} \int_{0}^{\tau_{j-1}} \cdots \int_{0}^{\tau_{1}} x\left(\tau_{j}\right) d \tau d \tau_{2} d \tau_{1}, \\
& x(t) \equiv x^{(0)}(t)=\sum_{i=1}^{k} t^{\alpha-i} x^{(\alpha-i)}(0) \\
& +a \int_{0}^{t} \int_{0}^{\tau_{k-1}} \cdots \int_{0}^{\tau_{1}} x\left(\tau_{k}\right) d \tau d \tau_{2} d \tau_{1} .
\end{aligned}
$$

Note that the solution is positive for any order $\alpha=k$ if $a \geq 0$ and $x^{(i)}(0) \geq 0$ for $i=1,2, \ldots, k$ and $x^{(0)}(0)=x(0)=x_{0}$. Assume that, for $a<0$, there is some $t>0$ such that $x(t)=0$ and $x_{k-1}(\tau)<0$ for $\tau \in(t, t+T)$ and some $T>0$ since it follows from continuity arguments that if it is negative at a point it is also negative on some interval containing the point. Then, the following contradiction arises:

$$
\begin{aligned}
0> & x(t+T) \\
= & \sum_{i=1}^{\alpha} T^{k-i} x^{(i)}(t) \\
& +|a| \int_{0}^{T} \int_{0}^{\tau_{k-1}} \cdots \int_{0}^{\tau_{1}}\left|x\left(\tau_{k}+t\right)\right| d \tau d \tau_{2} d \tau_{1} \\
\geq & |a| \int_{0}^{T} \int_{0}^{\tau_{\alpha-1}} \cdots \int_{0}^{\tau_{1}}\left|x\left(\tau_{k}+t\right)\right| d \tau d \tau_{2} d \tau_{1}>0
\end{aligned}
$$

so that any Caputo fractional solution of any real order $\alpha$ is nonnegative for $t \geq 0$ and any given initial conditions $x^{(i)}(0) \geq 0$ for $i=0,1, \ldots, k-1$. Since any solution is nonnegative for any nonnegative initial conditions, then if 
$a<0$, one gets for the case of interest $x^{(i)}(0)=0$ for $i=1,2, \ldots, k$

$$
\begin{aligned}
0 \leq & x(t) \\
= & \sum_{i=1}^{k} t^{k-i} x^{(k-i)}(0) \\
& \quad-|a| \int_{0}^{t} \int_{0}^{\tau_{k-1}} \cdots \int_{0}^{\tau_{1}} x\left(\tau_{k}\right) d \tau d \tau_{2} d \tau_{1} \\
= & x(0)-|a| \int_{0}^{t} \int_{0}^{\tau_{k-1}} \cdots \int_{0}^{\tau_{1}} x\left(\tau_{k}\right) d \tau d \tau_{2} d \tau_{1} \\
& \Longrightarrow \lim _{t \rightarrow \infty} x(t)=0
\end{aligned}
$$

and then global (resp., global asymptotic) stability holds for $a \leq 0$ (resp., for $a<0$ ) while the solutions are nonnegative for any nonnegative initial conditions and any real $a$. These stability properties are independent of the nonnegativity of the solutions since if $x(0)<0$ then by continuity of the solutions $x(t)<0$ in some interval $\left[0, t_{e}\right)$ so that

$$
\begin{array}{r}
x(t)=-|x(0)|+|a| \int_{0}^{t} \int_{0}^{\tau_{k-1}} \ldots \int_{0}^{\tau_{1}}\left|x\left(\tau_{k}\right)\right| d \tau d \tau_{2} d \tau_{1} \\
t \in\left[0, t_{e}\right)
\end{array}
$$

and $t_{e}=t_{e}\left(x_{0}\right)$ is large enough, since $r(t)=$ $\int_{0}^{t} \int_{0}^{\tau_{k-1}} \ldots \int_{0}^{\tau_{1}}\left|x\left(\tau_{k}\right)\right| d \tau d \tau_{2} d \tau_{1}$ is strictly increasing since it possesses a nonnegative integrand, so that there is $t^{*} \in\left[0, t_{e}\right)$ such that $x\left(t^{*}\right)=0$ and then $x(t)=0$; for all $t \geq t^{*}$, that is, the solution reaches the zero-equilibrium in finite time and global asymptotic stability is guaranteed. To evaluate the error between the $q$-calculus solution and the standard one, denote them, respectively, as $x(t)=x^{*}(t)+e(t)$ and $x^{*}(t)$ and then define the extended vector $v(t)=\left(x^{*}(t), e(t)\right)^{T}$. The substitution of both solutions yields

$$
v(t)=\left[\begin{array}{cc}
e^{a(1-q) t} & 0 \\
\frac{(1-q) a t}{1-(1-q) a t} & \frac{1}{1-(1-q) a t}
\end{array}\right] v(q t) ; \quad \forall t \in \mathbf{R}_{0+} .
$$

Note that for $a \neq 0$, the first eigenvalue of the matrix of dynamics tends to $+\infty$ if $a>0$ and to zero if $a<0$ while the second one converges to zero as $t \rightarrow \infty$ in both cases. Thus, the error between both solutions converges asymptotically to zero if $a \neq 0$. If $a=0$ then $v(t)=v(q t)$, for all $t \in \mathbf{R}_{0+}$.

\section{Preliminaries on Fractional $q$-Differential Systems}

Fundamental definitions of $q$-calculus are [35-38]

$$
[a]_{q}=\frac{1-q^{n}}{1-q}, \quad a \in \mathbf{R}, q \in(0,1) .
$$

The $q$-power function is

$$
\begin{array}{r}
(a-b)^{(0)}=1, \quad(a-b)^{(z)}=a^{z} \prod_{k=0}^{\infty}\left[\frac{a-b q^{k}}{a-b q^{z+k}}\right], \\
z(\in \mathbf{R}) \neq 0 ; \quad a, b \in \mathbf{R} .
\end{array}
$$

If $z \in \mathbf{Z}_{+}$then the $q$-power function is

$$
(a-b)^{(0)}=1, \quad(a-b)^{(z)}=\prod_{k=0}^{z-1}\left[a-b q^{k}\right]
$$

leading, in particular, to $(a-b)^{(1)}=\prod_{k=0}^{0}\left[a-b q^{k}\right]=a-$ $b$. Formula (16) is the $q$-analog of the Pochhammer symbol ( $q$-shifted factorial) [36]. The $q$-derivative of a function $f$ is defined by

$$
\begin{aligned}
& \left(D_{q} f\right)(z)=\frac{f(q z)-f(z)}{(q-1) z}, \\
& \left(D_{q} f\right)(0)=\lim _{z \rightarrow 0}\left(D_{q} f\right)(z)
\end{aligned}
$$

$[35,36]$ and the $q$-derivative of high $n$-order of a function $f$ is defined by $\left(D_{q}^{0} f\right)(z)=f(z)$ and

$$
\left(D_{q}^{n} f\right)(z)=D_{q}\left(D_{q}^{n-1} f\right)(z), \quad n \in \mathbf{Z}_{0+} .
$$

Lemma 3. The following properties hold.

(i) The commutation property $D_{q}^{j}\left(D_{q}^{k} f\right)(z)=$ $D_{q}^{k}\left(D_{q}^{j} f\right)(z) ;$ for all $j, k \in \mathbf{Z}_{0+}$, for all $q \in(0,1)$ holds for any real $z \neq 0$.

(ii) The commutation property $D_{q}^{j}\left(D_{q}^{k} f\right)(z)=$ $D_{q}^{k}\left(D_{q}^{j} f\right)(z) ;$ for all $j, k \in \mathbf{Z}_{0+}$, for all $q \in(0,1)$ holds for $z=0$ for any $j, k, n \in \mathbf{Z}_{0+}$, such that $n=j+k$, provided that $\left(D_{q}^{i} f\right)(0)$ exists for any $i \in \bar{n}=\{1,2, \ldots, n\}$.

(iii) $\left(D_{q}^{n}(f+g)\right)(z)=\left(D_{q}^{n} f\right)(z)+\left(D_{q}^{n} g\right)(z)$ or any real $z \neq 0$, and also for $z=0$ if $\left(D_{q}^{i} f\right)(0)$ exists for any $i \in \bar{n}=\{1,2, \ldots, n\}$.

(iv)

$$
\left(D_{q}^{n} f\right)(z)=\frac{1}{(1-q)^{n} z^{n}} \sum_{j=0}^{n}(-1)^{j}\left(\begin{array}{c}
n \\
j
\end{array}\right) f\left(q^{j} z\right)
$$

for all $n \in \mathbf{Z}_{0+}$, for all $q \in(0,1)$ holds for any real $z \neq 0$ and also for $z=0$ if

$$
\left(D_{q}^{n} f\right)(0)=\frac{1}{(1-q)^{n}} \lim _{z \rightarrow 0}\left(\sum_{j=0}^{n}(-1)^{j}\left(\begin{array}{l}
k \\
j
\end{array}\right) \frac{f\left(q^{j} z\right)}{z^{n}}\right)
$$

exist for all $n \in \mathbf{Z}_{0+}$.

Proof. First, note that for $z \neq 0$ the identity $\left(D_{q}^{n} f\right)(z)=$ $D_{q}\left(D_{q}^{n-1} f\right)(z)$ always holds for $n \in \mathbf{Z}_{0+}$, with $\left(D_{q}^{0} f\right)(z)=$ 
$f(z)$, which ensures the existence of $\left(D_{q}^{n} f\right)(z)$ for $n \in \mathbf{Z}_{0+}$ and $z \neq 0$. Proceed now by complete induction and assume that, for some given $k \in \mathbf{Z}_{0+}$, the commutation property below holds for the existing $\left(D_{q}^{n} f\right)(z)$ for any real $z \neq 0$ :

$$
\left(D_{q}^{n} f\right)(z)=D_{q}^{n-1}\left(D_{q} f\right)(z)=D_{q}\left(D_{q}^{n-1} f\right)(z) .
$$

Then, by using the commutation property of the operator $\left(D_{q} D_{q}^{n-1}\right)$ on $D_{q} f$ for any real $z \neq 0$

$$
\begin{aligned}
\left(D_{q} D_{q}^{n-1}\right)\left(D_{q} f\right)(z) & =\left(D_{q}^{n-1} D_{q}\right)\left(D_{q} f\right)(z) \\
& =\left(D_{q} D_{q}^{n} f\right)(z)=\left(D_{q}^{n} D_{q} f\right)(z) \\
& =\left(D_{q}^{n+1} f\right)(z) .
\end{aligned}
$$

The commutation of the operator composition $D_{q}^{n} D_{q}$ for any nonzero $z \in \mathbf{Z}_{+}$has been proven. Now, for any integer $z \geq 2$ we can find integers $j, k \in \mathbf{Z}_{+}$(being nonunique for $z \geq 3$ ) such that $j+k=n$. Hence, $\left(D_{q}^{j} D_{q}^{k} f\right)(z)=\left(D_{q}^{k} D_{q}^{j} f\right)(z)=$ $\left(D_{q}^{j+k} f\right)(z)$ and the result follows by taking an arbitrary $n$ and nonzero $z \in \mathbf{Z}_{+}$. Property (i) has been proved. Property (ii) can be proved in the same way for $j+k=n$ and any $n \in \mathbf{Z}_{0+}$ such that $\left(D_{q}^{i} f\right)(0)$ exists for $i \in \bar{n}=\{1,2, \ldots, n\}$. Properties (i)-(iii) have been proved.

On the other hand, the q-derivative operator (17) is a linear operator [37], so that

$$
\left(D_{q}(f+g)\right)(z)=\left(D_{q} f\right)(z)+\left(D_{q} g\right)(z) .
$$

Property (i) and (23) yield for $z \neq 0$, and also for $z=0$ if $\left(D_{q}^{i} f\right)(0)$ exists for any $i \in \bar{n}=\{1,2, \ldots, n\}$, the following relationships:

$$
\begin{aligned}
\left(D_{q}^{n}(f+g)\right)(z) & =\left(D_{q}\left(D_{q}^{n-1}(f+g)\right)\right)(z) \\
& =\left(D_{q}^{n-1}\left(D_{q}(f+g)\right)\right)(z) \\
& =\left(D_{q}^{n-1}\left(\left(D_{q} f\right)(z)+\left(D_{q} g\right)\right)\right)(z) \\
& \left.=D_{q}\left(\left(D_{q}^{n-1} f\right)(z)+\left(D_{q}^{n-1} g\right)\right)\right)(z) \\
& =\left(D_{q}^{n} f\right)(z)+\left(D_{q}^{n} g\right)(z) .
\end{aligned}
$$

Hence, Property (iii) follows.

To prove Property (iv), define the time $q$-delay operator $\mathbf{z}_{q}$ on $f$ as $\left(\mathbf{z}_{q} f\right)(z)=f(q z)$ so that $\left(\mathbf{z}_{q}^{j} f\right)(z)=f\left(q^{i} z\right)$, for all $j \in \mathbf{Z}_{0+}$ with $\left(\mathbf{z}_{q}^{0} f\right)(z)=f(z)$. Now, for any nonzero real $z$, assume that the property is true for $n=0,1, \ldots, k$ and some given $k \in \mathbf{Z}_{+}$. Thus,

$$
\begin{aligned}
\left(D_{q}^{n} f\right)(z) & =\frac{1}{(1-q)^{n} z^{n}} \sum_{j=0}^{n}(-1)^{j}\left(\begin{array}{c}
n \\
j
\end{array}\right) f\left(q^{j} z\right) \\
& =\frac{\left(\left(1-\mathbf{z}_{q}\right)^{n} f\right)(z)}{(1-q)^{n} z^{n}}
\end{aligned}
$$

for $n=0,1, \ldots, k$ from (19) (first identity) and the definition of the operator $\mathbf{z}_{q}$ (second identity). Then, one gets from the definition of the $q$-derivative in (17) the use of the first and second identities of (25) for $\left(D_{q}^{n} f\right)(z)$, with $n=k$, the identity $\left(\mathbf{z}_{q} f\right)(z)=f(q z)$, and, finally, the second identity in (25) for $n=k+1$ :

$$
\begin{aligned}
\left(D_{q}^{k+1} f\right)(z) & =\left(D_{q}\left(D_{q}^{k+1} f\right)\right)(z) \\
& =\frac{\left(\left(1-\mathbf{z}_{q}\right)^{k} f\right)(z)-\left(\left(1-\mathbf{z}_{q}\right)^{k} f\right)(q z)}{(1-q)^{k} z^{k}(1-q) z} \\
& =\frac{\left(\left(1-\mathbf{z}_{q}\right)^{k} f\right)(z)-\left(\left(1-\mathbf{z}_{q}\right)^{k} \mathbf{z}_{q} f\right)(z)}{(1-q)^{k+1} z^{k+1}} \\
& =\frac{\left(\left(1-\mathbf{z}_{q}\right)^{k+1} f\right)(z)}{(1-q)^{k+1} z^{k+1}} \\
& =\frac{1}{(1-q)^{k+1} z^{k+1}} \sum_{j=0}^{k+1}(-1)^{j}\left(\begin{array}{c}
k+1 \\
j
\end{array}\right) f\left(q^{j} z\right)
\end{aligned}
$$

so that if (19) holds for any given $\operatorname{real} z \neq 0$ and $n=0,1, \ldots, k$ for any given $k \in \mathbf{Z}_{+}$then it also holds for $n=0,1, \ldots, k+1$ and such a nonzero real $z$. Then, by complete induction, (19) is true for any nonnegative integer $n$. If $\left(D_{q}^{n} f\right)(0)$ exists for any $n \in \mathbf{Z}_{0+}$ the result also applies for $z=0$.

Assume that $f:[a, b] \rightarrow \mathbf{C}^{n}$ for some real interval $[a, b] \subset \mathbf{R}$. Then, the Riemann-Liouville left-sided fractional $q$-derivative ${ }^{\mathrm{RL}} D_{q a+}^{\alpha} f$ of order $\alpha \in \mathrm{C}_{0+}$ of the vector function $f$ in $[a, b]$ is point-wise defined as

$$
\begin{aligned}
& \left({ }^{\mathrm{RL}} D_{q a+}^{\alpha} f\right)(t) \\
& \quad:=\frac{1}{\Gamma_{q}(k-\alpha)}\left(D_{q}^{k}\left(\int_{a}^{t} \frac{f(\tau)}{(t-q \tau)^{\alpha+1-k}} d \tau_{q}\right)\right) \\
& t \in[a, b]
\end{aligned}
$$

where $k=[\operatorname{Re} \alpha]+1$ and $\Gamma: \mathbf{C} \backslash \mathbf{Z}_{0_{-}} \rightarrow \mathbf{C}$, where $\mathbf{Z}_{0-}:=\{n \in \mathbf{Z}: n \leq 0\}$, is the $q$-gamma function defined as $\Gamma_{q}(z):=(1-q)^{(z-1)} /(1-q)^{z-1}=\left(1 /(1-q)^{z-1}\right) \prod_{j=0}^{\infty}[(1-$ $\left.\left.q^{j}\right) /\left(1-q^{z+j-1}\right)\right] ; z \in \mathbf{C} \backslash \mathbf{Z}_{0-}$ which satisfies the following relations $[35,36]$ :

$$
\Gamma_{q}(z+1)=(1-q)^{(z)}(1-q)^{-z}=[z]_{q} \Gamma_{q}(z)
$$

where $[z]_{q}=\left(1-q^{z}\right) /(1-q)$ for $z \in \mathbf{R}$ is a $q$-real number. Remember that the usual gamma function is defined by $\Gamma(z):=\int_{0}^{\infty} \tau^{z-1} e^{-\tau} d \tau ; z \in \mathbf{C} \backslash \mathbf{Z}_{0-}$. Now, we can replace the standard simple and higher-order derivatives under the integral symbol by their $q$-derivative versions to 
build the Caputo fractional $q$-derivative ${ }^{C} D_{q a+}^{\alpha} f$ by using the identities

$$
\begin{aligned}
\left(D_{q}^{k} f\right)(\tau) & =f_{q}^{(k)}(\tau)=\frac{d_{q}^{k} f(\tau)}{d \tau_{q}^{k}} \\
& =D_{q}\left(D_{q}^{k-1} f\right)(\tau)=D_{q}^{k-1}\left(D_{q} f\right)(\tau)
\end{aligned}
$$

in $[a, b]$; then the Caputo left-sided fractional $q$-derivative ${ }^{C} D_{q a+}^{\alpha} f$ of order $\alpha \in \mathbf{C}_{0+}$ of the vector function $f$ in $[a, b] \subset$ $\mathbf{R}_{+}$is point-wise defined as

$$
\begin{array}{r}
\left({ }^{C} D_{q a+}^{\alpha} f\right)(t) \\
=\frac{1}{\Gamma_{q}(k-\alpha)} \int_{a}^{t}(t-q \tau)^{k-\alpha-1}\left(D_{q}^{k} f(\tau)\right) d_{q} \tau ; \\
t \in[a, b],
\end{array}
$$

where $k=[\operatorname{Re} \alpha]+1$ if $\alpha \notin \mathbf{Z}_{0+}$ and $k=\alpha$ if $\alpha \in \mathbf{Z}_{0+}$. Note that the existence of $f \in C^{k-1}\left((a, b), \mathbf{R}^{n}\right)$ in (30) is not required, as it is required in the standard fractional calculus for the existence of Caputo derivatives since the existence of the standard and higher-order fractional $q$-derivatives is ensured.

Example 4. Consider the differential dynamic system

$$
\dot{x}(t)=\sum_{i=0}^{p} A_{i}\left(t-h_{i}\right)+B u(t) ; \quad t \in[0, \infty),
$$

where $0=h_{0}<h_{1}<h_{2}<\cdots<h_{p}=h<\infty$ are distinct constant delays, $A_{0}, A_{i} \in \mathbf{R}^{n \times n}$; for all $i \in \bar{p}$ are the matrices of dynamics for each delay $h_{i}, i \in \bar{p} \cup\{0\}, B \in \mathbf{R}^{n \times m}$ is the control matrix and with initial condition of the state being given the real vector function $\varphi:[-h, 0] \rightarrow \mathbf{R}^{n}$, which is absolutely continuous except eventually in a set of zero measure of $[-h, 0] \subset \mathbf{R}$ of bounded discontinuities with $\varphi(0)=x(0)=x_{0} j \in \bar{p}$, and $u: \mathbf{R}_{0+} \rightarrow \mathbf{R}^{m}$ is a bounded piecewise-continuous control function. Some results about the nonnegativity of the solutions of (31) by using standard, fractional and $q$-calculus follow below.

Proposition 5. The solution of (31) is nonnegative on $[0, \infty)$ for any $\varphi:[-h, 0] \rightarrow \mathbf{R}_{0+}^{n}$ and $u: \mathbf{R}_{0+} \rightarrow \mathbf{R}_{0+}^{m}$ if and only if, $A_{i} \in \mathbf{R}_{0+}^{n \times n}$ for all $i \in \bar{p}$ and $A_{0} \in M \mathbf{R}^{n \times n}$.

Proof. The sufficiency part of the proof is direct since the unique left-sided mild solution is given by

$$
\begin{aligned}
x(t)=e^{A_{0} t}\left(x_{0}\right. & +\sum_{i=1}^{p} \int_{0}^{h_{i}} e^{-A_{0} \tau} A_{i} \varphi\left(\tau-h_{i}\right) d \tau \\
& +\sum_{i=1}^{p} \int_{h_{i}}^{t} e^{-A_{0} \tau} A_{i} x\left(\tau-h_{i}\right) d \tau \\
& \left.+\int_{0}^{t} e^{-A_{0} \tau} B u(\tau) d \tau\right) ; \quad \forall t \in \mathbf{R}_{0+} .
\end{aligned}
$$

It turns out that, under the given assumptions, $x: \mathbf{R}_{0+} \rightarrow$ $\mathbf{R}_{0+}^{n}$ and then the solution is nonnegative; for all $t \in \mathbf{R}_{0+}$ since $e^{A_{0} t} \in \mathbf{R}_{0+}^{n \times n}$; for all $t \in \mathbf{R}_{0+}$ since $A_{0} \in M \mathbf{R}^{n \times n}$. On the other hand,

$$
\begin{gathered}
x_{i}(t)=e_{i}^{T} e^{A_{0} t} x_{0} \\
+\sum_{j=1}^{n}\left(\sum_{i=1}^{p} \int_{0}^{h_{i}} e_{i}^{T} e^{A_{0}(t-\tau)}\right. \\
\times\left(A_{i j} \varphi_{j}\left(\tau-h_{i}\right) d \tau\right. \\
\left.\left.+\sum_{i=1}^{p} \int_{h_{i}}^{t} e^{-A_{0} \tau} A_{i j} x_{j}\left(\tau-h_{i}\right) d \tau\right)\right) \\
+\sum_{j=1}^{m} \sum_{i=1}^{p} \int_{h_{i}}^{t} e_{i}^{T} e^{A_{0}(t-\tau)} B_{i j} u_{j}\left(\tau-h_{i}\right) d \tau, \\
\forall t \in \mathbf{R}_{0+}, \quad i \in \bar{n} .
\end{gathered}
$$

It is easy to see that it is always possible to get $x_{i}(t)<0$ by construction if any of the conditions $\varphi:[-h, 0] \rightarrow \mathbf{R}_{0+}^{n}$, $A_{i} \in \mathbf{R}_{0+}^{n \times n}$; for all $i \in \bar{p}, u: \mathbf{R}_{0+} \rightarrow \mathbf{R}_{0+}^{m}$, or $A_{0} \in$ $M \mathbf{R}^{n \times n} \Leftrightarrow e^{A_{0} t} \in \mathbf{R}_{+}^{n}$; for all $t \in \mathbf{R}_{0+}$ fails for some $\varphi$ : $[-h, 0] \rightarrow \mathbf{R}_{0+}^{n}$ and $u: \mathbf{R}_{0+} \rightarrow \mathbf{R}_{0+}^{m}$ by taking some large component in either the initial condition function or the control function corresponding to a negative entry of the matrix whose positivity condition fails.

Proposition 6 (Theorem 4.1(iii) of [32]). Any solution (37) to any Caputo fractional differential system of fractional order $\alpha \in \mathbf{C}_{0+}$ is nonnegative independent of the delays; that is, $x(t) \in \mathbf{R}_{0+}^{n}$ for all $t \in[-h, \bar{t}) \cap \mathbf{R}_{0+}$ for some $\bar{t} \in \mathbf{R}_{0+}$, for any set of delays satisfying $0=h_{0}<h_{1}<h_{2}<\cdots<h_{p} \leq h<\infty$ and any absolutely continuous functions of initial conditions $\varphi_{j}:[-h, 0] \rightarrow \mathbf{R}_{0+}^{n}$, for all $j \in \overline{k-1} \cup\{0\}$ and any piecewise continuous control $u: \mathbf{R}_{0_{+}} \rightarrow \mathbf{R}_{0+}^{m}$, if and only if $A_{0} \in M \mathbf{R}^{n \times n}$ for $\bar{t} \in \mathbf{R}_{0+}$ being sufficiently small. Furthermore, $x(t) \in \mathbf{R}_{0+}^{n}$ for all $t \in[-h, 0) \cup \mathbf{R}_{0+}$ either if, in addition, $A_{0} \geq 0$ or if $A_{0}$ is nilpotent or if $0<\alpha \leq k=1, A_{i} \in \mathbf{R}_{0+}^{n \times n}$ ( for all $i \in \bar{p}$ ) and $B \in \mathbf{R}_{0+}^{n \times m}$.

Proposition 7. Consider the q-calculus version of (31) under similar initial conditions:

$$
\begin{array}{r}
\left(D_{q} x\right)(t):=\frac{x(t)-x(q t)}{(1-q) t}=\sum_{i=0}^{p} A_{i}\left(t-h_{i}\right)+B u(t) ; \\
t \in[0, \infty) .
\end{array}
$$

Assume that $\varphi:[-h, 0] \rightarrow \mathbf{R}_{0+}^{n}, u: \mathbf{R}_{0+} \rightarrow \mathbf{R}_{0+}^{m}, A_{i} \in$ $\mathbf{R}_{0+}^{n \times n}$ for all $i \in \bar{p}$. Then,

(i) $x(t) \geq 0$; for all $t \in \mathbf{R}_{0+}$ if either $1>A_{0 i i}>0$ or $A_{0 i i} \leq 0$ and $A_{0 i j}=0$ for all $i, j(\neq i) \in \bar{n}$. 
(ii) $x(t) \geq 0$; forall $t \in \mathbf{R}_{0+}$ if $\left(-A_{0}\right) \in M \mathbf{R}_{+}^{n \times n}$ with $A_{0 i i}=$ -1 ; forall $i \in \bar{n}$ and $A_{0 i j} \leq 0$ with $A_{0 i j}<0$ for only one $j \in \bar{n}$ for each $i \in \bar{n}$.

Proof. The solution of (34) is

$$
\begin{array}{r}
x(t)=\left(I-A_{0}\right)^{-1}\left(x(q t)+(1-q) t \sum_{i=1}^{p} A_{i} x\left(t-h_{i}\right)\right) \\
\forall t \in \mathbf{R}_{0+}
\end{array}
$$

provided that $\left(I-A_{0}\right)^{-1}$ exists. Since $\varphi:[-h, 0] \rightarrow \mathbf{R}_{0+}^{n}$, $u: \mathbf{R}_{0+} \rightarrow \mathbf{R}_{0+}^{m}, A_{i} \in \mathbf{R}_{0+}^{n \times n}$, for nonnegativity of the solutions the existence of $\left(I-A_{0}\right)^{-1} \in \mathbf{R}_{+}^{n \times n}$ is also required. Note that if $\left(I-A_{0}\right) \in \mathbf{R}_{+}^{n \times n}$ and nonsingular then $\left(I-A_{0}\right)^{-1} \in \mathbf{R}_{+}^{n \times n}$ if and only if $\left(I-A_{0}\right) \in \mathbf{R}_{+}^{n \times n}$ is monomial (or generalized permutation matrix, i.e., it has only a nonzero entry per row and then only a nonzero entry per column). Due to its structure, this condition can be fulfilled with $A_{0}$ being diagonal, that is, $A_{0 i j}=0$ for $i, j(\neq i) \in \bar{n}$ in order that $\left(I-A_{0}\right)$ is monomial and $\left(I-A_{0}\right) \in \mathbf{R}_{+}^{n \times n}$. Then, $\left(I-A_{0}\right)^{-1} \in$ $\mathbf{R}_{0+}^{n \times n}$ if and only if for all $i \in \bar{n}$ either $1>A_{0 i i}>0$ or $A_{0 i i} \leq 0$. Hence, Property (i) follows directly. Property (ii) follows since, under the given constraints, $\left(-A_{0}\right) \in M \mathbf{R}^{n \times n}$, with $A_{0 i i}=-1$ for all $i \in \bar{n},\left(I-A_{0}\right) \in \mathbf{R}_{+}^{n \times n}$ and Metzler, monomial (then nonsingular) so that $\left(I-A_{0}\right)^{-1} \in \mathbf{R}_{+}^{n \times n}$. Hence, Property (ii) follows.

The following extension of Proposition 7 is obvious.

Proposition 8. Proposition 7 still holds if $A_{i}: \mathbf{R}_{0+} \rightarrow \mathbf{R}^{n \times n}$; $i \in \bar{n} \cup\{0\}$.

\section{Fractional $q$-Differential Dynamic Systems of Order $\alpha$ with Internal Point Delays}

The fractional Caputo $q$-differential dynamic systems of order $\alpha$ can become modified from (2). The functions $\Gamma_{q}(k-\alpha)$, $D_{q}^{k}\left(\int_{a}^{t}(t-q \tau)^{k-\alpha-1} f(\tau) d \tau_{q}\right), \quad$ and $\quad \int_{a}^{t}(t-q \tau)^{k-\alpha-1}\left(D_{q}^{k} f\right)$ $(\tau) d_{q} \tau$ are now displayed to be used later in order to solve such a differential system. The combination of (2) with (24) with the replacement of $f(t)$ with $x(t)$ leads to

$$
\begin{gathered}
\frac{1}{\Gamma_{q}(k-\alpha)} \int_{a}^{t}(t-q \tau)^{k-\alpha-1}\left(D_{q}^{k} x\right)(\tau) d_{q} \tau \\
\quad=\sum_{i=0}^{p} A_{i} x\left(t-h_{i}\right)+B u(t) ; \quad t \in[a, b] .
\end{gathered}
$$

(a) If $\alpha \in \mathbf{R}_{+}$and $\alpha \notin \mathbf{Z}_{0+}$ then $k<\alpha+1$, and

$$
\begin{aligned}
\frac{1}{\Gamma_{q}(k-\alpha)} & =\frac{(1-q)^{k-\alpha-1}}{(1-q)^{(k-\alpha-1)}} \\
& =(1-q)^{k-\alpha-1} \prod_{j=0}^{\infty}\left[\frac{1-q^{k+j-\alpha}}{1-q^{j+1}}\right] .
\end{aligned}
$$

(b) If $\alpha \in \mathbf{Z}_{0+}$ then $k=\alpha$, and

$$
\frac{1}{\Gamma_{q}(0)}=\frac{1-q}{(1-q)^{(1)}}=1 \text {. }
$$

Now consider, from (2) and (24), the linear time-varying differential functional left-sided Caputo fractional $q$-differential system of order $\alpha$

$$
\begin{aligned}
\left({ }^{C} D_{q 0+}^{\alpha} x\right)(t)= & (1-q)^{k-\alpha-1} \prod_{j=0}^{\infty}\left[\frac{1-q^{k+j-\alpha}}{1-q^{j+1}}\right] \\
& \times \int_{a}^{t}(t-q \tau)^{k-\alpha-1}\left(D_{q}^{k} x\right)(\tau) d_{q} \tau \\
= & \sum_{i=0}^{p} A_{i}(t) x\left(t-h_{i}\right)+B(t) u(t)
\end{aligned}
$$

with $k-1<\alpha\left(\in \mathbf{R}_{+}\right) \leq k ; k-1, k \in \mathbf{Z}_{0+}, 0=h_{0}<h_{1}<$ $h_{2}<\cdots<h_{p}=h<\infty$ being distinct constant delays; $A_{0}$ : $\mathbf{R}_{0+} \rightarrow \mathbf{R}^{n}, A_{i}: \mathbf{R}_{0+} \rightarrow \mathbf{R}^{n}(i \in \bar{p}:=\{1,2, \ldots, p\})$, are the bounded matrix functions of dynamics for each delay $h_{i}, \quad i \in$ $\bar{p} \cup\{0\}$, and $B: \mathbf{R}_{0+} \rightarrow \mathbf{R}^{n \times m}$ is the control matrix function. The initial conditions are given by $k n$-real vector functions $\varphi_{j}:[-h, 0] \rightarrow \mathbf{R}^{n}$, with $j \in \overline{k-1} \cup\{0\}$ and with $\varphi_{j}(0)=$ $x_{j}(0)=x^{(j)}(0)=x_{j 0}$, and $u: \mathbf{R}_{0+} \rightarrow \mathbf{R}^{m}$ is a bounded piecewise continuous control function. The Jackson integral in the integral term in (39) becomes

$$
\begin{aligned}
& \int_{a}^{t}(t-q \tau)^{k-\alpha-1}\left(D_{q}^{k} x\right)(\tau) d_{q} \tau \\
& =\int_{0}^{t}(t-q \tau)^{k-\alpha-1}\left(D_{q}^{k} x\right)(\tau) d_{q} \tau \\
& \quad-\int_{0}^{a}(t-q \tau)^{k-\alpha-1}\left(D_{q}^{k} x\right)(\tau) d_{q} \tau \\
& \quad-(1-q) a \sum_{n=0}^{\infty}\left(1-q^{n+1}\right)^{k-\alpha-1} a^{k-\alpha-1} q^{n}\left(D_{q}^{k} x\right)\left(q^{n} a\right)
\end{aligned}
$$

for $t \geq a$. By replacing the dummy argument $\tau$ under the two integral symbols in (40) by $q^{n} t$ and $q^{n} a$, respectively, since (see [37]):

$$
\int_{0}^{t} f(\tau) d_{q} \tau=(1-q) t \sum_{n=0}^{\infty} f\left(q^{n} t\right) q^{n}
$$

The series in (41) is convergent for all $t \in \mathbf{R}_{0+}$ since the function $f$ satisfies $|f(t)|<B t^{C}$ in a right neighborhood of $t=0$ for some real constants $B>0$ and $C>-1$. So, the Jackson integral in (40) is convergent since $\left\|\left(D_{q}^{k} x\right)(t)\right\|<$ $B t^{C+\alpha+1-k}$ in some right neighbourhood of $t=0$ for real 
constants $B>0$ and $C>-1[37,39]$. Equations (40) and (39), together with (18), Lemma 3 (see (19)), and (17), yield:

$$
\begin{aligned}
& \left({ }^{C} D_{q a+}^{\alpha} x\right)(t) \\
& =(1-q)^{k-\alpha-1} \prod_{j=0}^{\infty}\left[\frac{1-q^{k+j-\alpha}}{1-q^{j+1}}\right] \\
& \times(1-q)\left[t \sum_{n=0}^{\infty}\left(1-q^{n+1}\right)^{k-\alpha-1} t^{k-\alpha-1} q^{n}\right. \\
& \times D\left(\left(D_{q}^{k-1} x\right)\right)\left(q^{n} t\right)-a \sum_{n=0}^{\infty}\left(1-q^{n+1}\right)^{k-\alpha-1} \\
& \left.\quad \times a^{k-\alpha-1} q^{n} D\left(\left(D_{q}^{k-1} x\right)\right)\left(q^{n} a\right)\right] \\
& =\sum_{i=0}^{p} A_{i}(t) x\left(t-h_{i}\right)+B(t) u(t) .
\end{aligned}
$$

The following auxiliary results hold concerning the product $\prod_{j=0}^{n}\left[\left(1-q^{k+j-\alpha}\right) /\left(1-q^{j+1}\right)\right]$ and the series $\sum_{n=0}^{\infty}\left(1-q^{n+1}\right)^{k-\alpha-1} t^{k-\alpha-1} q^{n} D\left(\left(D_{q}^{k-1} x\right)\right)\left(q^{n} t\right)$.

Lemma 9. Define $P_{n}:=\left(\prod_{j=0}^{n}\left[\left(1-q^{k+j-\alpha}\right) /\left(1-q^{j+1}\right)\right]\right)$. Then, $P_{n+1}=g_{n} P_{n}$ for all $n \in \mathbf{Z}_{0+}$, where

(a) $P_{0}=0$ and $g_{n}=1$, for all $n \in \mathbf{Z}_{0+}$, so that $P_{n} \equiv 0$, if $\alpha \in \mathbf{Z}_{0+}$;

(b) $P_{0}=\left(1-q^{k-\alpha}\right) /(1-q)=(k-\alpha)_{q}$ and $g_{n}:=$ $(k+n+1-\alpha)_{q} / \sum_{i=0}^{n} q^{i}<1$, for all $n \in \mathbf{Z}_{0+}$, if $\alpha \notin$ $\mathrm{Z}_{0+}$;

(c) $\exists \lim _{n \rightarrow \infty} P_{n}=P^{*}$.

Proof. Note that

(1) $P_{n+1} / P_{n}=\left(\left(1-q^{k+n+1-\alpha}\right) /\left(1-q^{n+1}\right)\right) /\left(\left(1-q^{k+n-\alpha}\right) /(1-\right.$ $\left.\left.q^{n}\right)\right) \rightarrow 1$ as $n \rightarrow \infty$;

(2) if $k=\alpha\left(\in \mathbf{Z}_{0+}\right), P_{0}=0, \quad P_{1}=0, P_{n+1} / P_{n}=1$ y $P=$ $P_{n}=P_{0}=(k-\alpha)_{q}=[k-\alpha]_{q}=[0]_{q}=0 ;$

(3) if $k \neq \alpha\left(\in \mathbf{R}_{+}\right)$, with $\alpha \notin \mathbf{Z}_{0+}$, then $P_{0}=$ (1 $\left.q^{k-\alpha}\right) /(1-q)=(k-\alpha)_{q}, P_{1}=\left(\left(1-q^{k-\alpha}\right) /(1-q)\right)((1-$ $\left.\left.q^{k+1-\alpha}\right) /\left(1-q^{2}\right)\right)=(1 /(1+q))(k-\alpha)_{q}(k+1-\alpha)_{q}=$ $\left((k+1-\alpha)_{q} /(1+q)\right) P_{0}, P_{2}=\left(\left(1-q^{k+2-\alpha}\right) /(1-\right.$ $\left.\left.q^{3}\right)\right) P_{1}=\left(1 /\left(1+q+q^{2}\right)\right)\left(\left(1-q^{k+2-\alpha}\right) /(1-q)\right) P_{1}=$ $\left(1 /\left(1+q+q^{2}\right)\right)(k+2-\alpha)_{q} P_{1}, P_{n+1}=g_{n} P_{n}$ with $g_{n}=$ $(k+n+1-\alpha)_{q} / \sum_{i=0}^{n} q^{i}$ and $g_{n}<1$. Assume this is false so that $g_{n} \geq 1$. Then, $1-q^{n}-q^{k+n+1-\alpha}+q^{k+2 n+1-\alpha} \geq$ $1-q^{n+1}-q^{k+n-\alpha}+q^{k+2 n+1-\alpha}$ and, equivalently, $\left(-q^{n}-\right.$ $\left.q^{k+n+1-\alpha}\right) \geq\left(-q^{n+1}-q^{k+n-\alpha}\right)$, or $q^{n}(1-q) \leq q^{k+n-\alpha}(1-$ q) $\Rightarrow 1 \leq q^{k-\alpha}<1$ if $\alpha<k<\alpha+1$ (a contradiction).
Lemma 10. Assume that $\left\|\left(D_{q}^{k} x\right)(t)\right\| t^{k+\gamma-\alpha-1}$ is bounded in a right neighborhood $\left[0, t_{0}\right)$ of $t=0$ for some $t_{0} \in \mathbf{R}_{+}$and some real constant $1>\gamma \geq 0$. Define

$$
S(t, n):=\sum_{i=0}^{n}\left(1-q^{i+1}\right)^{k-\alpha-1} t^{k-\alpha} q^{i}\left(D_{q^{k}}^{k}\right)\left(q^{i} t\right)
$$

$\forall t \in \mathbf{R}_{0+}, \quad \forall n \in \mathbf{Z}_{0+}$.

Then, the following identities hold:

$$
\begin{array}{r}
\sum_{i=0}^{n}\left(1-q^{i+1}\right)^{k-\alpha-1} t^{k-\alpha} q^{i}\left[\left(D_{q}^{k} x\right)\left(q^{i+1} t\right)-\left(D_{q}^{k} x\right)\left(q^{i} t\right)\right] \\
=q^{\alpha-k}(S(q t, n)-S(t, n)) ; \quad \forall t \in \mathbf{R}_{0+}, \forall n \in \mathbf{Z}_{0+},
\end{array}
$$

$$
\begin{gathered}
\sum_{i=0}^{\infty}\left(1-q^{i+1}\right)^{k-\alpha-1} t^{k-\alpha} q^{i}\left[\left(D_{q}^{k} x\right)\left(q^{i+1} t\right)-\left(D_{q}^{k} x\right)\left(q^{i} t\right)\right] \\
=q^{\alpha-k}\left(S^{*}(q t)-S^{*}(t)\right) ; \quad \forall t \in\left[0, t_{0}\right),
\end{gathered}
$$

$$
\begin{aligned}
S^{*}(t)= & \frac{1}{(1-q)^{\alpha+1} t^{\alpha}} x(t)+\widehat{S}_{N t}\left(\widehat{q}_{N} t\right)+\widetilde{S}_{N t}\left(\widetilde{q}_{N} t\right), \\
\widehat{S}_{N t}\left(\widehat{q}_{N} t\right)= & \frac{1}{(1-q)^{\alpha+1} t^{\alpha}} \sum_{j=1}^{k}(-1)^{j}\left(\begin{array}{c}
k \\
j
\end{array}\right) x\left(q^{j} t\right) \\
& +\sum_{i=1}^{N} \sum_{j=0}^{k}(-1)^{j} \frac{q^{i}}{\left(1-q^{i+1}\right)(1-q)^{k} t^{\alpha}} \\
& \times\left(\begin{array}{c}
k \\
j
\end{array}\right) x\left(q^{j+i} t\right),
\end{aligned}
$$

$\widetilde{S}_{N t}\left(\widetilde{q}_{N} t\right)=\sum_{i=N+1}^{\infty} \sum_{j=0}^{k}(-1)^{j} \frac{q^{i}}{\left(1-q^{i+1}\right)(1-q)^{k} t^{\alpha}}\left(\begin{array}{c}k \\ j\end{array}\right) x\left(q^{j+i} t\right)$

for any given $N \in \mathbf{Z}_{0+}$ if $\alpha \notin \mathbf{Z}_{0+}$, where $k=[\operatorname{Re} \alpha]+1$, $\widehat{q}_{N}=\left(q, q^{2}, \ldots, q^{N+k}\right), \widetilde{q}_{N}=\left(q^{N+1}, q^{N+2}, q^{N+3}, \ldots\right), S^{*}(t)=$ $\lim _{n \rightarrow \infty} S(t, n)$; for all $t \in \mathbf{R}_{0+}$, since such a limit function exists; for all $t \in \mathbf{R}_{0+}$, and $\widetilde{S}_{N t}(t) \rightarrow 0$ as $N \rightarrow \infty$; for all $t \in \mathbf{R}_{0+}$

$$
\begin{aligned}
\left|\widetilde{S}_{N t}\left(\widetilde{q}_{N} t\right)\right| \leq & \left(\frac{\gamma}{q^{k}}\right)^{N+1} \frac{(k+1)\left(\|x(0)\|+\varepsilon_{N}\right)}{(1-\gamma)(1-q)^{k} t^{\alpha}} \\
& \times\left|\sum_{j=0}^{k}(-1)^{j}\left(\begin{array}{c}
k \\
j
\end{array}\right)\right|,
\end{aligned}
$$


where $\left\{\varepsilon_{N}\right\}$ is a nonnegative real sequence which converges to $0 ; \gamma \in \mathbf{R}_{+}$is subject to

$$
1>\gamma>q \max \left(1, \max _{i \geq N+1} \frac{1}{\left(1-q^{i+1}\right)^{(1+\alpha-k) / i}}\right)
$$

for any given $\mathrm{N} \in \mathbf{Z}_{0+}$.

Equations (46)-(47) also hold for $k=\alpha\left(\in \mathbf{Z}_{0+}\right)$ by replacing $k \rightarrow \alpha$ everywhere $k$ appears. Consider

$$
\begin{array}{r}
S(t, n+1)=S(t, n)+d(t, n)\left(D_{q}^{k} x\right)\left(q^{n+1} t\right) \\
\forall t \in \mathbf{R}_{0+}, \quad \forall n \in \mathbf{Z}_{0+}, \\
\exists \lim _{n \rightarrow \infty}\left[\left(1-q^{n+2}\right)^{k-\alpha-1} t^{k-\alpha} q^{n+1}\left(D_{q}^{k} x\right)\left(q^{n+1} t\right)\right]=0 ; \\
\forall t \in \mathbf{R}_{0+},
\end{array}
$$

where $d(t, n)=\left(1-q^{n+2}\right)^{k-\alpha-1} t^{k-\alpha} q^{n+1}$.

Proof. Since $\left\|\left(D_{q}^{k} x\right)(t)\right\| t^{k+\gamma-\alpha-1}$ is bounded in a right neighbourhood $\left[0, t_{0}\right)$ of $t=0$ for some real constant $1>\gamma \geq 0$ then the Jackson integral

$$
\begin{aligned}
\int_{0}^{t}(t & -q \tau)^{k-\alpha-1}\left(D_{q}^{k} x\right)(\tau) d_{q} \tau \\
& =(1-q) \sum_{n=0}^{\infty}\left(1-q^{n+1}\right)^{k-\alpha-1} t^{k-\alpha} q^{n}\left(D_{q}^{k} x\right)\left(q^{n} t\right)
\end{aligned}
$$

converges to a function $F(t)$ on $\left[0, t_{0}\right)$ which is a $q$ antiderivative of $(t-q \tau)^{k-\alpha-1}\left(D_{q}^{k} x\right)(\tau)$ and which is continuous at $t=0$ with $F(0)=0$ and is a unique $(t-q \tau)^{k-\alpha-1}\left(D_{q}^{k} x\right)(\tau)$ in this class of functions [39]. Note that $S(t, n) \leq S^{*}(t)=\lim _{n \rightarrow \infty} S(t, n)$; for all $n \in \mathbf{Z}_{0+} ; t \in\left[0, t_{0}\right)$ since the series $S(t, n)$ is convergent, since the Jackson integral (52) is convergent for each fix $t \in\left[0, t_{0}\right)$, and non-decreasing with $n$ since it is consists of nonnegative terms. It follows from (43), with the replacement $t \rightarrow q t$, that

$$
\begin{aligned}
S(q t, n)= & q^{k-\alpha}\left[\sum_{i=0}^{n}\left(1-q^{i+1}\right)^{k-\alpha-1} t^{k-\alpha} q^{i}\left(D_{q}^{k} x\right)\left(q^{i+1} t\right)\right] \\
= & q^{k-\alpha}(S(t, n) \\
+ & \sum_{i=0}^{n}\left(1-q^{i+1}\right)^{k-\alpha-1} t^{k-\alpha} q^{i} \\
& \left.\times\left[\left(D_{q}^{k} x\right)\left(q^{i+1} t\right)-\left(D_{q}^{k} x\right)\left(q^{i} t\right)\right]\right) ;
\end{aligned}
$$

for all $t \in\left[0, t_{0}\right)$ and hence (44) follows. By taking limits as $n \rightarrow \infty$ in (44), one gets (46)-(48) since the limit $S^{*}(t)=\lim _{n \rightarrow \infty} S(t, n)$ exists for all $t \in \mathbf{R}_{0+}$ by using Lemma 3(iv) for the expansion of the $q$-derivative of order $k$ since $q^{n} \rightarrow 0$ as $n \rightarrow \infty$. Equation (49) follows by defining $\gamma_{i}:=\left(1-q^{i+1}\right)^{(k-\alpha-1) / i} q<1$ with $\gamma_{i}^{i} \leq \gamma^{i}<1$ for some $1>\gamma>q$; for all $i(\geq N) \in \mathbf{Z}_{0+}$ and some $N \in \mathbf{Z}_{0+}$, or equivalently

$$
\left(1-q^{i+1}\right)^{1+a-k} \geq\left(\frac{q}{\gamma}\right)^{i} \Longleftrightarrow 1 \geq q^{i+1}+\left(\frac{q}{\gamma}\right)^{i /(1+a-k)}
$$

Hence, (46)-(48) follow from (55) for some strictly decreasing real sequence $\left\{\varepsilon_{N}\right\} \in \mathbf{R}_{+}$such that $\left|x\left(q^{j+i} t\right)\right| \leq|x(0)|+\varepsilon_{N}$; for all $i(\geq N) \in \mathbf{Z}_{0+}$ for $j=0,1, \ldots, k$.

The quantum Caputo fractional solutions have explicit expressions as formulated in the subsequent result.

Theorem 11. Consider the left-sided q-fractional Caputo solution of or fractional order $\alpha$ of the functional differential system

$$
\left({ }^{C} D_{q 0+}^{\alpha} x\right)(t)=\sum_{i=0}^{p} A_{i}(t) x\left(t-h_{i}\right)+B(t) u(t) ; \quad t \in[0, \infty)
$$

with initial condition of the state $x:[-h, \infty) \rightarrow \mathbf{R}^{n}$ being defined by $k$ n-real vector functions $\varphi_{j}:[-h, 0] \rightarrow \mathbf{R}^{n}$, with $j \in \overline{k-1} \cup\{0\}$ and $\varphi_{j}(0)=x_{j}(0)=x^{(j)}(0)=x_{j 0} j \in \overline{k-1} \cup$ $\{0\}$, and $u: \mathbf{R}_{0+} \rightarrow \mathbf{R}^{m}$ is a bounded piecewise continuous control function and $h=h_{p}=\max _{1 \leq i \leq p} h_{i}$.

Thus, the unique left-sided solution of (55) is calculated almost everywhere via analytical expressions obtained from Lemma 10 as follows.

(i) If $\alpha \notin \mathbf{Z}_{0+}$ so that $k=[\operatorname{Re} \alpha]+1$ then one gets for any given $N \in \mathbf{Z}_{+}$that

$$
\begin{aligned}
x(t)= & \left(\frac{\left(P_{N}+\rho_{N}\right)}{(1-q)^{2 \alpha-k+1} t^{\alpha}} I-A_{0}(t)\right)^{-1} \\
& \times\left(\sum_{j=1}^{p} A_{j}(t) x\left(t-h_{j}\right)-\left(P_{N}+\rho_{N}\right)(1-q)^{k-\alpha}\right. \\
& \left.\times\left[\widehat{S}_{N t}\left(\widehat{q}_{N} t\right)+\widetilde{S}_{N t}\left(\widetilde{q}_{N} t\right)\right]+B u(t)\right) ;
\end{aligned}
$$

for all $t \in T R_{N K}$, where $T R_{N k}:=\left\{t \in \mathbf{R}_{+}:\left(\left(\left(P_{N}+\right.\right.\right.\right.$ $\left.\left.\left.\rho_{N}\right) /(1-q)^{2 \alpha-k+1} t^{\alpha}\right) I-A_{0}(t)\right)$ is non singular $\}$. 
(ii) If $k=\left(\alpha \in \mathbf{Z}_{0+}\right)$ then one gets for any given $N \in \mathbf{Z}_{+}$ that

$$
\begin{aligned}
x(t)= & \left(\frac{1}{(1-q)^{\alpha+1} t^{\alpha}} I-A_{0}(t)\right)^{-1} \\
& \times\left(\sum_{j=1}^{p} A_{j}(t) x\left(t-h_{j}\right)-\widehat{S}_{N t}\left(\widehat{q}_{N} t\right)\right. \\
= & \left.\left(\frac{1}{(1-q)^{\alpha+1} t^{\alpha}} I-A_{0}(t)\right)^{-1}\left(\widetilde{q}_{N} t\right)+B(t) u(t)\right) \\
& \times\left(\sum_{j=1}^{p} A_{j}(t) x\left(t-h_{j}\right)\right. \\
& \left.+\frac{1}{(1-q)^{\alpha+1} t^{\alpha}} x(t)-S^{*}(t)+B(t) u(t)\right) ;
\end{aligned}
$$

for all $t \in T R_{\alpha}$, where $T R_{\alpha}:=\left\{t \in \mathbf{R}_{+}\right.$: $\left(\left(1 /(1-q)^{\alpha+1} t^{\alpha}\right) I-A_{0}(t)\right)$ is nonsingular $\}$.

Proof. One can evaluate the approximation of (42) for $a=0$ and $\alpha \notin \mathbf{Z}_{0+}$ by using (46) as

$$
\begin{aligned}
\left({ }^{C} D_{q 0+}^{\alpha} x\right)(t)= & \left(P_{N}+\rho_{N}\right)(1-q)^{k-\alpha} \\
& \times\left[\frac{1}{(1-q)^{\alpha+1} t^{\alpha}} x(t)+\widehat{S}_{N t}\left(\widehat{q}_{N} t\right)\right. \\
& \left.\quad \widetilde{S}_{N t}\left(\widetilde{q}_{N} t\right)\right] \\
= & \sum_{j=0}^{p} A_{j}(t) x\left(t-h_{j}\right)+B(t) u(t) \quad \text { if } \alpha \notin \mathbf{Z}_{0+},
\end{aligned}
$$

where

$$
\begin{array}{r}
\rho_{N}:=P^{*}-P_{N} \longrightarrow 0, \quad \widetilde{S}_{N t}\left(\widetilde{q}_{N} t\right) \longrightarrow 0 \\
\text { as } N \longrightarrow \infty ; \quad \forall t \in \mathbf{R}_{0+}
\end{array}
$$

with $\rho_{N} \rightarrow 0, \sigma_{N}(t) \rightarrow 0$ as $N \rightarrow \infty$, for all $t \in \mathbf{R}_{0+}$. On the other hand, for $a=0$ and $k=\alpha\left(\in \mathbf{Z}_{0+}\right)$, note that $1 / \Gamma(0)=(1-q)^{k-\alpha}\left(\prod_{j=0}^{\infty}\left[\left(1-q^{k+j-\alpha}\right) /\left(1-q^{j+1}\right)\right]\right)=P_{N}+$
$\rho_{N}=1$ from (37)-(38) so that (58) (with $k=\alpha$ ) becomes simplified as follows:

$$
\begin{aligned}
& \left({ }^{C} D_{q 0+}^{\alpha} x\right)(t) \\
& \quad=\left[\frac{1}{(1-q)^{\alpha+1} t^{\alpha}} x(t)+\widehat{S}_{N t}\left(\widehat{q}_{N} t\right)+\widetilde{S}_{N t}\left(\widetilde{q}_{N} t\right)\right] \\
& \quad=\sum_{j=0}^{p} A_{j}(t) x\left(t-h_{j}\right)+B(g) u(t) \quad \text { if } k=\left(\alpha \in \mathbf{Z}_{0+}\right) .
\end{aligned}
$$

Thus, the (unique) left-sided solution of (55) follows directly from (59) and (60).

A variant of Theorem 11 might be obtained in a simple way by selecting the initial point $a=a(t)=t-\zeta$ so that the real interval $[t-a(t), t]$ has a constant measure for each $t(\geq h)$ $\epsilon \mathbf{R}_{+}$independent of $t$. For that purpose, one implements the quantum fractional left-sided Caputo derivative with the operator $\left({ }^{C} D_{q(t-\zeta)+}^{\alpha} x\right)(t)$ replacing $\left({ }^{C} D_{q 0+}^{\alpha} x\right)(t)$ while fixing $a(t)=t-\zeta$ in (42) to reformulate (55) with the appropriate "ad hoc" modifications of (56) and (57).

Theorem 12. Consider the left-sided q-fractional Caputo solution of or fractional order $\alpha \in \mathbf{R}_{0+}$ of the functional differential system

$$
\begin{aligned}
& \left({ }^{C} D_{q(t-\zeta)+}^{\alpha} x\right)(t) \\
& \quad=\sum_{i=0}^{p} A_{i}(t)\left(t-h_{i}\right)+B u(t) ; \quad t \in[0, \infty)
\end{aligned}
$$

for some prefixed $\zeta \in \mathbf{R}_{+}$, with initial condition of the state $x:[-h, \infty) \rightarrow \mathbf{R}^{n}$ being defined by $k$ n-real vector functions $\varphi_{j}:[-h, 0] \rightarrow \mathbf{R}_{0+}^{n}$, with $j \in \overline{k-1} \cup\{0\}$, which are bounded with $\varphi_{j}(0)=x_{j}(0)=x^{(j)}(0)=x_{j 0} j \in \overline{k-1} \cup\{0\}$, and $u: \mathbf{R}_{0+} \rightarrow \mathbf{R}^{m}$ is a bounded piecewise continuous control function and $h=h_{p}=\max _{1 \leq i \leq p} h_{i}$.

Thus, the unique left-sided solution of (55) is calculated almost everywhere via analytical expressions as follows.

(i) If $\alpha \notin \mathbf{Z}_{0+}$ so that $k=[\operatorname{Re} \alpha]+1$ then one gets for any given $N \in \mathbf{Z}_{+}$that

$$
\begin{aligned}
x(t)= & \left(\frac{\left(P_{N}+\rho_{N}\right)}{(1-q)^{2 \alpha-k+1} t^{\alpha}} I-A_{0}(t)\right)^{-1} \\
& \times\left(\sum_{j=1}^{p} A_{j}(t) x\left(t-h_{j}\right)+B(t) u(t)\right. \\
& +\left(P_{N}+\rho_{N}\right)(1-q)^{k-\alpha} \\
& \times\left(\widehat{S}_{N(t-\zeta)}\left(\widehat{q}_{N}(t-\zeta)\right)\right. \\
& \left.\left.+\widetilde{S}_{N(t-\zeta)}\left(\widetilde{q}_{N}(t-\zeta)\right)-\widehat{S}_{N t}\left(\widehat{q}_{N} t\right)-\widetilde{S}_{N t}\left(\widetilde{q}_{N} t\right)\right)\right) ;
\end{aligned}
$$


forall $t \in T R_{N K}$, where $T R_{N k}:=\left\{t \in \mathbf{R}_{+}:\left(\left(\left(P_{N}+\right.\right.\right.\right.$ $\left.\left.\left.\rho_{N}\right) /(1-q)^{2 \alpha-k+1} t^{\alpha}\right) I-A_{0}(t)\right)$ is nonsingular $\}$, where $\widehat{S}_{N(t-\zeta)}\left(\widehat{q}_{N} t\right)$ and $\widetilde{S}_{N(t-\zeta)}\left(\widetilde{q}_{N} t\right)$ are got by replacing $t \rightarrow$ $(t-\zeta)$ in their counterparts $\widehat{S}_{N t}\left(\widehat{q}_{N} t\right)$ and $\widetilde{S}_{N t}\left(\widetilde{q}_{N} t\right)$ defined in (47)-(48).

(ii) If $k=\left(\alpha \in \mathbf{Z}_{0_{+}}\right)$then one gets for any given $N \in \mathbf{Z}_{+}$that

$$
\begin{aligned}
x(t)= & \left(\frac{1}{(1-q)^{\alpha+1} t^{\alpha}} I-A_{0}(t)\right)^{-1} \\
& \times\left(\sum_{j=1}^{p} A_{j}(t) x\left(t-h_{j}\right)+B(t) u(t)\right. \\
& \quad+\widehat{S}_{N(t-\zeta)}\left(\widehat{q}_{N}(t-\zeta)\right)+\widetilde{S}_{N(t-\zeta)} \\
& \left.\quad \times\left(\widetilde{q}_{N}(t-\zeta)\right)-\widehat{S}_{N t}\left(\widehat{q}_{N} t\right)-\widetilde{S}_{N t}\left(\widetilde{q}_{N} t\right)\right) ;
\end{aligned}
$$

forall $t \in T R_{\alpha}$, where $T R_{\alpha}:=\left\{t \in \mathbf{R}_{+}\right.$: $\left(\left(1 /(1-q)^{\alpha+1} t^{\alpha}\right) I-A_{0}(t)\right)$ is nonsingular $\}$, where $\widehat{S}_{N(t-\zeta)}\left(\widehat{q}_{N} t\right)$ and $\widetilde{S}_{N(t-\zeta)}\left(\widetilde{q}_{N} t\right)$ are got by replacing $t \rightarrow$ $(t-\zeta)$ in their counterparts $\widehat{S}_{N t}\left(\widehat{q}_{N} t\right)$ and $\widetilde{S}_{N t}\left(\widetilde{q}_{N} t\right)$ defined in (47)-(48).

Proof. It is omitted since it follows directly as that of Theorem 11 by using (42) with $a=a(t)=t-\zeta$, for all $t \in$ $\mathbf{R}_{0+}$.

The following auxiliary result is useful to then formulate the solution calculation under a finite truncation of the infinite series associated with the Jackson integral.

Lemma 13. The following properties hold.

(i) For any $\varepsilon \in \mathbf{R}_{+}$and any finite real $T_{1}>\varepsilon$ and $T_{2}>\zeta+\varepsilon$, the limits below exist:

$$
\begin{gathered}
\lim _{N \rightarrow \infty} \widetilde{S}_{N t}\left(\widetilde{q}_{N} t\right)=0 ; \quad t \in\left[\varepsilon, T_{1}\right), \\
\lim _{N \rightarrow \infty}\left[\widetilde{S}_{N t}\left(\widetilde{q}_{N} t\right)-\widetilde{S}_{N(t-\zeta)}\left(\widetilde{q}_{N}(t-\zeta)\right)\right]=0 ; \\
t \in\left[\zeta+\varepsilon, T_{2}\right) .
\end{gathered}
$$

(ii) Assume, furthermore, that the solution $x: \mathbf{R}_{0+} \rightarrow \mathbf{R}^{n}$ of (61) has the following property:

$$
\limsup _{t \rightarrow \infty}\left(\sup _{i \geq N}\left\|\frac{x\left(q^{i} t\right)}{t^{\alpha}}-\frac{x\left(q^{i}(t-\zeta)\right)}{(t-\zeta)^{\alpha}}\right\|-O(t-\zeta)^{\alpha}\right)=0
$$

Then, the limit below exists:

$$
\lim _{t \rightarrow \infty} \lim _{N \rightarrow \infty}\left[\widetilde{S}_{N t}\left(\widetilde{q}_{N} t\right)-\widetilde{S}_{N(t-\zeta)}\left(\widetilde{q}_{N}(t-\zeta)\right)\right]=0
$$

Proof. One gets from Lemma 10, (48) that

$$
\begin{aligned}
& \left\|\widetilde{S}_{N(t-\zeta)}\left(\widetilde{q}_{N}(t-\zeta)\right)-\widetilde{S}_{N t}\left(\tilde{q}_{N} t\right)\right\| \\
& \leq \| \sum_{i=N+1}^{\infty} \sum_{j=0}^{k}(-1)^{j} \frac{q^{i}}{\left(1-q^{i+1}\right)(1-q)^{k}}\left(\begin{array}{c}
k \\
j
\end{array}\right) \\
& \quad \times\left(\frac{x\left(q^{j+i}(t-\zeta)\right)}{(t-\zeta)^{\alpha}}-\frac{x\left(q^{j+i} t\right)}{t^{\alpha}}\right) \| \\
& \leq 2 \frac{q^{N+1}}{(1-q)^{k+1}\left(1-q^{N+1}\right)} \quad \forall t(\geq \zeta) \in \mathbf{R}_{0+}
\end{aligned}
$$

since

$$
\begin{aligned}
\sum_{i=N+1}^{\infty} \frac{q^{i}}{1-q^{i+1}} & \leq \sum_{i=N+1}^{\infty} \frac{q^{i}}{1-q^{i}} \\
& \leq \sum_{i=N+1}^{\infty} \frac{q^{i}}{1-q^{N+1}} \leq \frac{q^{N+1}}{(1-q)\left(1-q^{N+1}\right)}
\end{aligned}
$$

In a similar way, one can obtain

$$
\begin{aligned}
\left\|\widetilde{S}_{N t}\left(\widetilde{q}_{N} t\right)\right\| \leq & \frac{q^{N+1}}{(1-q)^{k+1}\left(1-q^{N+1}\right)} \\
& \times \sup _{i \geq N}\left[\frac{1}{t^{\alpha}}\left(\left\|x\left(q^{j+i} t\right)\right\|\right)\right] ; \quad \forall t \in \mathbf{R}_{0+}
\end{aligned}
$$

and hence Property (i) follows since one has from (67) and (69) for some finite positive real constants $M_{1}=M\left(\varepsilon, T_{1}\right)$ and $M_{2}=M\left(\zeta+\varepsilon, T_{2}\right)$ that

$$
\begin{aligned}
\left\|\widetilde{S}_{N t}\left(\tilde{q}_{N} t\right)\right\| \leq & \frac{q^{N+1}}{(1-q)^{k+1}\left(1-q^{N+1}\right)} \\
& \times \sup _{\varepsilon \leq t \leq T_{1}} \sup _{i \geq N}\left[\frac{1}{\varepsilon^{\alpha}}\left(\left\|x\left(q^{j+i}(t)\right)\right\|\right)\right] \\
\leq & \frac{q^{N+1} M_{1}}{(1-q)^{k+1}\left(1-q^{N+1}\right)} ; \quad \forall t \in\left[\varepsilon, T_{1}\right), \\
\left\|\widetilde{S}_{N t}\left(\widetilde{q}_{N} t\right)-\widetilde{S}_{N(t-\zeta)}\left(\widetilde{q}_{N}(t-\zeta)\right)\right\| & \leq \frac{2 q^{N+1} M_{2}}{(1-q)^{k+1}\left(1-q^{N+1}\right)} ; \quad \forall t \in\left[\zeta+\varepsilon, T_{2}\right) .
\end{aligned}
$$


Note that if the solution of (61) satisfies (65) then

$$
\begin{aligned}
& \left\|\widetilde{S}_{N(t-\zeta)}\left(\widetilde{q}_{N}(t-\zeta)\right)-\widetilde{S}_{N t}\left(\widetilde{q}_{N} t\right)\right\| \\
& \leq \| \sum_{i=N+1}^{\infty} \sum_{j=0}^{k}(-1)^{j} \frac{q^{i}}{\left(1-q^{i+1}\right)(1-q)^{k}} \\
& \quad \times\left(\begin{array}{c}
k \\
j
\end{array}\right)\left(\frac{x\left(q^{j+i}(t-\zeta)\right)}{(t-\zeta)^{\alpha}}-\frac{x\left(q^{j+i} t\right)}{t^{\alpha}}\right) \| \\
& \leq \frac{K_{S} q^{N+1}}{1-q} \sup _{i \geq N}\left\|\left(\frac{x\left(q^{i}(t-\zeta)\right)}{(t-\zeta)^{\alpha}}-\frac{x\left(q^{i} t\right)}{t^{\alpha}}\right)\right\| .
\end{aligned}
$$

Then,

$$
\begin{aligned}
& \limsup _{t \rightarrow \infty}\left\|\widetilde{S}_{N(t-\zeta)}\left(\tilde{q}_{N}(t-\zeta)\right)-\widetilde{S}_{N t}\left(\tilde{q}_{N} t\right)\right\| \\
& \quad \leq \frac{K_{S} q^{N+1}}{1-q} \limsup _{t \rightarrow \infty}\left(\sup _{i \geq N}\left\|\left(\frac{x\left(q^{i}(t-\zeta)\right)}{(t-\zeta)^{\alpha}}-\frac{x\left(q^{i} t\right)}{t^{\alpha}}\right)\right\|\right)
\end{aligned}
$$

so that

$$
\begin{aligned}
0 & \leq \limsup _{t \rightarrow \infty} \lim _{N \rightarrow \infty}\left\|\widetilde{S}_{N(t-\zeta)}\left(\widetilde{q}_{N}(t-\zeta)\right)-\widetilde{S}_{N t}\left(\widetilde{q}_{N} t\right)\right\| \\
& \leq\left(\lim _{N \rightarrow \infty} \frac{K_{S} q^{N+1}}{1-q}\right) \limsup _{t \rightarrow \infty}\left(0(t-\zeta)^{\alpha}\right)=0
\end{aligned}
$$

and then $\exists \lim _{t \rightarrow \infty} \lim _{N \rightarrow \infty}\left\|\widetilde{S}_{N(t-\zeta)}\left(\widetilde{q}_{N}(t-\zeta)\right)-\widetilde{S}_{N t}\left(\widetilde{q}_{N} t\right)\right\|=$ 0 . Hence, Property (ii) follows.

The subsequent result follows directly from Theorem 12 and Lemma 13.

Theorem 14. The following properties hold for $\alpha \in \mathbf{R}_{0+}$.

(i) If $\alpha \notin \mathbf{Z}_{0+}$ so that $k=[\operatorname{Re} \alpha]+1$ then one gets

$$
\begin{gathered}
\lim _{N \rightarrow \infty} \| x(t)-\left(\frac{P^{*}}{(1-q)^{2 \alpha-k+1} t^{\alpha}} I-A_{0}(t)\right)^{-1} \\
\times\left(\sum_{j=1}^{p} A_{j} x\left(t-h_{j}\right)+B(t) u(t)\right. \\
+P^{*}(1-q)^{k-\alpha}\left(\widehat{S}_{N(t-\zeta)}\left(\widehat{q}_{N}(t-\zeta)\right)\right. \\
\left.\left.-\widehat{S}_{N t}\left(\widehat{q}_{N} t\right)\right)\right) \|=0 ; \\
t \in \bar{T} \bar{R}_{k \alpha}
\end{gathered}
$$

$$
\text { for any real } \varepsilon \in \mathbf{R}_{+} \text {and } \zeta+\varepsilon \leq T<\infty \text {, where }
$$

$$
\begin{aligned}
\bar{T}_{k \alpha}:= & \{t \in[\zeta+\varepsilon, T): \\
& \left.\left(\frac{P^{*}}{(1-q)^{2 \alpha-k+1} t^{\alpha}} I-A_{0}(t)\right) \text { is nonsingular }\right\} .
\end{aligned}
$$

If, in addition, $A_{0}$ is nonsingular and $\limsup _{t \rightarrow \infty}\left(\sup _{i \geq N} \| x\left(q^{i} t\right) / t^{\alpha} \quad-\quad x\left(q^{i}(t \quad-\right.\right.$ $\left.\zeta)) /(t-\zeta)^{\alpha} \|-O(t-\zeta)^{\alpha}\right)=0$ then

$$
\begin{aligned}
\lim _{t \rightarrow \infty} \lim _{N \rightarrow \infty} \| x(t)+A_{0}^{-1}(t) \\
\quad \times\left(\sum_{j=1}^{p} A_{j}(t) x\left(t-h_{j}\right)+B(t) u(t)\right. \\
\quad+P^{*}(1-q)^{k-\alpha}\left(\widehat{S}_{N(t-\zeta)}\left(\widehat{q}_{N}(t-\zeta)\right)\right.
\end{aligned}
$$

$$
\left.-\widehat{S}_{N t}\left(\widehat{q}_{N} t\right)\right) \|=0 \text {. }
$$

(ii) If $k=\left(\alpha \in \mathbf{Z}_{0+}\right)$ then one gets

$$
\begin{aligned}
\lim _{N \rightarrow \infty} \| x(t) & -\left(\frac{1}{(1-q)^{\alpha+1} t^{\alpha}} I-A_{0}(t)\right)^{-1} \\
& \times\left(\sum_{j=1}^{p} A_{j}(t) x\left(t-h_{j}\right)+B u(t)\right. \\
& \left.+\widehat{S}_{N(t-\zeta)}\left(\widehat{q}_{N}(t-\zeta)\right)-\widehat{S}_{N t}\left(\widehat{q}_{N} t\right)\right) \|=0 ; \\
& \quad t \in \bar{T}_{\alpha}
\end{aligned}
$$

for any real $\varepsilon \in \mathbf{R}_{+}$and $\zeta+\varepsilon \leq T<\infty$, where

$$
\begin{aligned}
\overline{T R}_{\alpha}:= & \{t \in[\zeta+\varepsilon, T): \\
& \left.\left(\frac{1}{(1-q)^{\alpha+1} t^{\alpha}} I-A_{0}(t)\right) \text { is nonsingular }\right\} .
\end{aligned}
$$


If, in addition, $A_{0}$ is nonsingular and $\lim \sup _{t \rightarrow \infty}$ $\left(\sup _{i \geq N}\left\|x\left(q^{i} t\right) / t^{\alpha}-x\left(q^{i}(t-\zeta)\right) /(t-\zeta)^{\alpha}\right\|-O(t-\zeta)^{\alpha}\right)=$ 0 then

$$
\begin{aligned}
\lim _{t \rightarrow \infty} \lim _{N \rightarrow \infty} \| x(t)+A_{0}^{-1}(t) & \\
& \times\left(\sum_{j=1}^{p} A_{j}(t) x\left(t-h_{j}\right)+B(t) u(t)+\widehat{S}_{N(t-\zeta)}\right. \\
& \left.\quad \times\left(\widehat{q}_{N}(t-\zeta)\right)-\widehat{S}_{N t}\left(\widehat{q}_{N} t\right)\right) \|=0 .
\end{aligned}
$$

\section{Nonnegativity of the $q$-Solutions of Caputo Fractional Order $\alpha$}

The following two results hold for the nonnegativity for all time of the solutions of (55) under certain nonnegative controls.

Theorem 15. Consider the Caputo fractional q-differential system of order $\alpha \in \mathbf{R}_{0+}$ (55) under a piecewise-continuous control vector function $u: \mathbf{R}_{0+} \rightarrow \mathbf{R}_{0_{+}}^{n}$ and any set of vector functions of initial conditions $\varphi_{j}:[-h, 0] \rightarrow \mathbf{R}_{0+}^{n}$, for all $j \in \overline{k-1} \cup\{0\}$. Assume also that $\alpha \notin \mathbf{Z}_{0+}, B(t)=$ $\left(b_{i j}(t)\right) \in \mathbf{R}_{+}^{n \times n}$ is a monomial diagonal matrix (i.e., $b_{i i}>$ 0 and $b_{i j}=0$; for all $\left.i, j(\neq i) \in \bar{n}\right)$ and that the control components satisfy the constraints for some $N \in \mathbf{Z}_{+}$and some real nonnegative strictly decreasing sequence $\left\{\varepsilon_{n}\right\}$ :

$$
\begin{aligned}
& u_{i}(t) \\
& \qquad \max \left(0, b_{i i}^{-1}(t)\right. \\
& \times\left(P_{N}+\rho_{N}\right)(1-q)^{k-\alpha} \\
& \quad \times\left[e_{i}^{T} \widehat{S}_{N t}\left(\widehat{q}_{N} t\right)-\left(\frac{\gamma}{q^{k}}\right)^{N+1}\right. \\
& \left.\quad \times \frac{(k+1)\left(\|x(0)\|+\varepsilon_{N}\right)}{(1-\gamma)(1-q)^{k} t^{\alpha}}\left|\sum_{j=0}^{k}(-1)^{j}\left(\begin{array}{c}
k \\
j
\end{array}\right)\right|\right] \\
& \left.\left.\quad \sum_{j=1}^{p} e_{i}^{T} A_{j} x\left(t-h_{j}\right)\right)\right)^{k} \quad \forall i \in \bar{n}, \forall t \in \mathbf{R}_{0+},
\end{aligned}
$$

where $e_{i}$ is the unit Euclidean vector of $\mathbf{R}^{n}$ with its ith component being one. Then, the solution of (55) is nonnegative, that is, $x:[-h, 0] \cup \mathbf{R}_{0+} \rightarrow \mathbf{R}_{0+}^{n}$ under a nonnegative real controlu : $\mathbf{R}_{0+} \rightarrow \mathbf{R}_{0+}^{n}$ if one of the following conditions holds.
(C1) Either $\left(P_{N}+\rho_{N}\right) /(1-q)^{2 \alpha-k+1} t^{\alpha}>A_{0 i i}(t)>0$ or $A_{0 i i}(t) \leq 0$ with $\limsup _{t \rightarrow \infty} A_{0 i i}(t)<0$ and $A_{0 i j}(t)=0$, for all $i, j(\neq i) \in \bar{n}$, for all $t \in \mathbf{R}_{0+}$ or

(C2) $\left(-A_{0}(t)\right) \in M \mathbf{R}_{+}^{n \times n}, A_{0 i i}(t)=-\left(P_{N}+\right.$ $\left.\rho_{N}\right) /(1-q)^{2 \alpha-k+1} t^{\alpha}$, for all $i \in \bar{n}, A_{0 i j}(t) \leq 0$ with $A_{0 i j}(t)<0$ for only one $j \in \bar{n}$ for each $i \in \bar{n}$, for all $t \in \mathbf{R}_{0+}$.

Proof. Since $\alpha \notin \mathbf{Z}_{0+}$ then $k=[\operatorname{Re} \alpha]+1$ and one gets from (55)-(58), (49), and (80) that

$$
\begin{aligned}
x(t) \geq & \left(\frac{P_{N}+\rho_{N}}{(1-q)^{2 \alpha-k+1} t^{\alpha}} I-A_{0}(t)\right)^{-1} \\
& \times\left(\sum_{j=1}^{p} A_{j}(t) x\left(t-h_{j}\right)-\left(P_{N}+\rho_{N}\right)(1-q)^{k-\alpha}\right. \\
& \times\left[\begin{array}{c}
\widehat{S}_{N t}\left(\widehat{q}_{N} t\right)+\left(\frac{\gamma}{q^{k}}\right)^{N+1} \frac{(k+1)\left(\|x(0)\|+\varepsilon_{N}\right)}{(1-\gamma)(1-q)^{k} t^{\alpha}} \\
\left.\left.\times\left|\sum_{j=0}^{k}(-1)^{j}\left(\begin{array}{c}
k \\
j
\end{array}\right)\right| \widehat{e}\right]+B(t) u(t)\right) \geq 0 ; \\
\quad \forall t \in \mathbf{R}_{0+}
\end{array}\right.
\end{aligned}
$$

since $\quad\left(\left(\left(P_{N}+\rho_{N}\right) /(1-q)^{2 \alpha-k+1} t^{\alpha}\right) I-A_{0}(t)\right)^{-1} \in$ $\mathbf{R}_{+}^{n \times n}$ for all $t \in \mathbf{R}_{0+}$ under any of conditions (C1) or (C2) where $\hat{e} \in \mathbf{R}^{n}$ is a real vector with all its components being one, since

$$
\begin{aligned}
& \left(\sum_{j=1}^{p} A_{j}(t) x\left(t-h_{j}\right)-\widehat{S}_{N t}\left(\widehat{q}_{N} t\right)+\left(\frac{\gamma}{q^{k}}\right)^{N+1}\right. \\
& \quad \times \frac{(k+1)\left(\|x(0)\|+\varepsilon_{N}\right)}{(1-\gamma)(1-q)^{k} t^{\alpha}}\left|\sum_{j=0}^{k}(-1)^{j}\left(\begin{array}{c}
k \\
j
\end{array}\right)\right| \widehat{e} \\
& +B(t) u(t)) \geq 0 ; \quad \forall t \in \mathbf{R}_{0+}
\end{aligned}
$$

from (80) and any of (C1)-(C2) implies the existence of $\left(\left(\left(P_{N}+\rho_{N}\right) /(1-q)^{2 \alpha-k+1} t^{\alpha}\right) I-A_{0}(t)\right)^{-1} \in \mathbf{R}_{+}^{n \times n} ;$ for all $t \epsilon$ $\mathbf{R}_{0+}$.

Theorem 16. Under similar assumptions for the control, the matrix $B$ and the initial conditions as in Theorem 15, assume 
that $\alpha=k \in \mathbf{Z}_{0+}, B(t)=\left(b_{i j}(t)\right) \in \mathbf{R}_{+}^{n \times n}$ and that the control components satisfy the constraints

$$
\begin{aligned}
& u_{i}(t) \geq \max \left(0, b_{i i}^{-1}(t)\right. \\
& \times\left(e_{i}^{T} \widehat{S}_{N t}\left(\widehat{q}_{N} t\right)-\left(\frac{\gamma}{q^{k}}\right)^{N+1}\right. \\
& \times \frac{(k+1)\left(\|x(0)\|+\varepsilon_{N}\right)}{(1-\gamma)(1-q)^{k} t^{\alpha}}\left|\sum_{j=0}^{\alpha}(-1)^{j}\left(\begin{array}{c}
\alpha \\
j
\end{array}\right)\right| \\
& \left.\left.\quad-\sum_{j=1}^{p} e_{i}^{T} A_{j}(t) x\left(t-h_{j}\right)\right)\right) ;
\end{aligned}
$$$$
\forall i \in \bar{n}, \quad \forall t \in \mathbf{R}_{0+}
$$

for some $\mathrm{N} \in \mathbf{Z}_{+}$and some real nonnegative strictly decreasing sequence $\left\{\varepsilon_{n}\right\}$. Then, $x:[-h, 0] \cup \mathbf{R}_{0+} \rightarrow \mathbf{R}_{0+}^{n}$ under $a$ nonnegative real control $u: \mathbf{R}_{0+} \rightarrow \mathbf{R}_{0+}^{n}$ if one of the following conditions holds.

(C3) Either $(1-q)^{k-\alpha} /(1-q)^{\alpha+1} t^{\alpha}>A_{0 i i}(t)>0$ or $A_{0 i i}(t) \leq 0$ with $\limsup _{t \rightarrow \infty} A_{0 i i}(t)<0$ and $A_{0 i j}(t)=$ ofor all $i, j(\neq i) \in \bar{n}$; for each $t \in \mathbf{R}_{0+}$, or

(C4) $\left(-A_{0}(t)\right) \in M \mathbf{R}_{+}^{n \times n}$ with $A_{0 i i}(t)=-(1-q)^{k-\alpha} /(1-$ $q)^{\alpha+1} t^{\alpha} ;$ for all $i \in \bar{n}$ and $A_{0 i j}(t) \leq 0$ with $A_{0 i j}(t)<0$ for only one $j \in \bar{n}$ for each $i \in \bar{n}$; for each $t \in \mathbf{R}_{0+}$.

Proof. Since $\alpha(=k) \in \mathbf{Z}_{0+}$, one gets from (55)-(58), (49), and (83) that

$$
\begin{gathered}
x(t) \geq\left(\frac{1}{(1-q)^{\alpha+1} t^{\alpha}} I-A_{0}(t)\right)^{-1} \\
\times\left(\sum_{j=1}^{p} A_{j}(t) x\left(t-h_{j}\right)-\left(P_{N}+\rho_{N}\right)(1-q)^{k-\alpha}\right. \\
\times\left[\widehat{S}_{N t}\left(\widehat{q}_{N} t\right)+\left(\frac{\gamma}{q^{k}}\right)^{N+1}\right. \\
\times \frac{(k+1)\left(\|x(0)\|+\varepsilon_{N}\right)}{(1-\gamma)(1-q)^{k} t^{\alpha}} \\
\left.\left.\times\left|\sum_{j=0}^{\alpha}(-1)^{j}\left(\begin{array}{c}
\alpha \\
j
\end{array}\right)\right| \widehat{e}\right]+B(t) u(t)\right) \geq 0 ;
\end{gathered}
$$

since there is $\left(\left(1 /(1-q)^{\alpha+1} t^{\alpha}\right) I-A_{0}(t)\right)^{-1} \in \mathbf{R}_{+}^{n \times n}$, for all $t \epsilon$ $\mathbf{R}_{0+}$ under any of conditions (C3) or (C4). Then,

$$
\begin{aligned}
x(t) \geq & \left(\frac{1}{(1-q)^{\alpha+1} t^{\alpha}} I-A_{0}(t)\right)^{-1} \\
& \times\left(\sum_{j=1}^{p} A_{j}(t) x\left(t-h_{j}\right)-\widehat{S}_{N t}\left(\widehat{q}_{N} t\right)\right. \\
& +B(t) u(t)-\left(\frac{\gamma}{q^{k}}\right)^{N+1} \frac{(\alpha+1)\left(\|x(0)\|+\varepsilon_{N}\right)}{(1-\gamma)(1-q)^{k} t^{\alpha}} \\
& \left.\times\left|\sum_{j=0}^{\alpha}(-1)^{j}\left(\begin{array}{c}
\alpha \\
j
\end{array}\right)\right| \hat{e}\right) \geq 0 ; \quad \forall t \in \mathbf{R}_{0+}
\end{aligned}
$$

from (83) and any of conditions (C3) or (C4) imply from Proposition 7 the existence of $\left(\left(1 /(1-q)^{\alpha+1} t^{\alpha}\right) I-A_{0}(t)\right)^{-1} \epsilon$ $\mathbf{R}_{+}^{n \times n}$, for all $t \in \mathbf{R}_{0+}$.

Remark 17. If the $q$-Caputo fractional differential system (61) is considered instead of (55), Theorems 15 and 16 apply with the replacements

$$
\begin{aligned}
&\left\|\widehat{S}_{N t}\left(\widetilde{q}_{N} t\right)\right\| \longrightarrow\left\|\widehat{S}_{N(t-\zeta)}\left(\widetilde{q}_{N}(t-\zeta)\right)-\widehat{S}_{N t}\left(\widetilde{q}_{N} t\right)\right\| ; \\
&\left\|\widetilde{S}_{N t}\left(\widetilde{q}_{N} t\right)\right\| \longrightarrow\left\|\widetilde{S}_{N(t-\zeta)}\left(\widetilde{q}_{N}(t-\zeta)\right)-\widetilde{S}_{N t}\left(\widetilde{q}_{N} t\right)\right\|
\end{aligned}
$$

for any given $\zeta \in \mathbf{R}_{+}$and for all $t(\geq \zeta) \in \mathbf{R}_{+}$with the second replacement leading to the replacement below in (80)-(85) from (67) so as to keep the validity of both theorems under the modified nonnegative controls (80) and (83), respectively:

$$
\begin{aligned}
& \left(\frac{\gamma}{q^{k}}\right)^{N+1} \frac{(k+1)\left(\|x(0)\|+\varepsilon_{N}\right)}{(1-\gamma)(1-q)^{k} t^{\alpha}} \\
& \quad \longrightarrow 2 \frac{q^{N+1}}{(1-q)^{k+1}\left(1-q^{N+1}\right)} \\
& \quad \times \sup _{i \geq N}\left[\frac{1}{(t-\zeta)^{\alpha}}\left(\left\|x\left(q^{j+i}(t-\zeta)\right)\right\|,\left\|x\left(q^{j+i} t\right)\right\|\right)\right] ; \\
& \forall t(\geq \zeta) \in \mathbf{R}_{0+} .
\end{aligned}
$$

Theorem 15 is extendable for a nondiagonal square or a rectangular control matrix as follows.

Theorem 18. Consider (55) with $\alpha \notin \mathbf{Z}_{0+}$ under a piecewisecontinuous control vector function $u: \mathbf{R}_{0+} \rightarrow \mathbf{R}_{0+}^{m}$, with $m \leq n$, and any set of vector functions of initial conditions $\varphi_{j}:[-h, 0] \rightarrow \mathbf{R}_{0+}^{n}$, for all $j \in \overline{k-1} \cup\{0\}$. Assume also that $B(t)=\left(b_{i j}(t)\right) \in \mathbf{R}_{+}^{n \times m}$ has positive rows $b_{i}^{T}(t)=\left(b_{i 1}(t), b_{i 2}(t), \ldots, b_{i m}(t)\right) \in \mathbf{R}_{+}^{n}$, that the control components of $u(t)$ are generated as $u_{j}(t)=\lambda_{j}(t) m(t)$, where 
$\lambda_{j}: \mathbf{R}_{0+} \rightarrow \mathbf{R}_{0+}$ and the solution nonnegativity lowerbounding function $m: \mathbf{R}_{0+} \rightarrow \mathbf{R}_{+}$satisfy the constraints $\inf _{t \in \mathbf{R}_{0+}} \min _{i \in \bar{n}}\left(\sum_{j=1}^{m} b_{i j}(t) \lambda_{j}(t)\right)>0$, and

$$
\begin{aligned}
m(t) \geq \max _{i \in \bar{n}}\{ & \left(\sum_{j=1}^{m} b_{i j}(t) \lambda_{j}(t)\right)^{-1} \\
& \times\left(P_{N}+\rho_{N}\right)(1-q)^{k-\alpha} \\
& \times\left[\left(\frac{\gamma}{q^{k}}\right)^{N+1} \frac{(k+1)\left(\|x(0)\|+\varepsilon_{N}\right)}{(1-\gamma)(1-q)^{k} t^{\alpha}}\right. \\
& \left.\times\left|\sum_{j=0}^{k}(-1)^{j}\left(\begin{array}{c}
k \\
j
\end{array}\right)\right|\right] \\
& \left.\left.\quad \sum_{j=1}^{p} e_{i}^{T} A_{j}(t) x\left(t-h_{j}\right)-e_{i}^{T} \widehat{S}_{N t}\left(\widehat{q}_{N} t\right)\right)\right\}
\end{aligned}
$$

for some $N \in \mathbf{Z}_{+}$and some nonnegative strictly decreasing sequence $\left\{\varepsilon_{n}\right\}$. Then, the solution of (55) is nonnegative under a nonnegative real control if either (C1) or (C2) of Theorem 15 holds.

The proof of Theorem 18 is direct and then omitted. Close extensions to Theorem 18 for Theorem $16\left(\alpha=k \in \mathbf{Z}_{0+}\right)$ and for Remark 17 (the Caputo fractional derivative operator is defined on finite sliding intervals) can be directly made for a nondiagonal square or a rectangular control matrix.

Theorem 19. Consider (55) under any set of bounded vector functions of initial conditions $\varphi_{j}:[-h, 0] \rightarrow \mathbf{R}_{0+}^{n}$, for all $j \in$ $\overline{k-1} \cup\{0\}$. Assume also that $B(t) \in \mathbf{R}_{+}^{n \times m}$, with $m \geq n$ and rank $B(t)=n$, for all $t \in \mathbf{R}_{0+}$, and that the following control law is used for some given bounded vector function $u_{0}: \mathbf{R}_{0+} \rightarrow$ $\mathbf{R}_{0+}^{m}$ :

$$
\begin{aligned}
& u(t)=B^{T}(t)\left(B(t) B^{T}(t)\right)^{-1} u_{1}(t), \\
& u_{1}(t)=u_{0}(t)-\sum_{j=1}^{p} A_{j}(t) x\left(t-h_{j}\right) \\
& +\left(P_{N}+\rho_{N}\right)(1-q)^{k-\alpha} \\
& \times\left[\widehat{S}_{N t}\left(\widehat{q}_{N} t\right)+\left(\frac{\gamma}{q^{k}}\right)^{N+1} \frac{(k+1)\left(\|x(0)\|+\varepsilon_{N}\right)}{(1-\gamma)(1-q)^{k} t^{\alpha}}\right. \\
& \left.\times\left|\sum_{j=0}^{k}(-1)^{j}\left(\begin{array}{l}
k \\
j
\end{array}\right)\right| \hat{e}\right] .
\end{aligned}
$$

(i) The solution of (55) is nonnegative and bounded if $\alpha \notin$ $\mathbf{Z}_{0+}$ and either condition (C1) or condition (C2) of Theorem 15 holds. If, in addition, $u_{0}(t) \rightarrow 0$ as $t \rightarrow \infty$ then the solution $x: \mathbf{R}_{0+} \rightarrow \mathbf{R}_{0+}^{m}$ of (55) converges asymptotically to $x_{e}=0$ so that (55) is globally asymptotically stable for any control law and any set of vector functions of initial conditions satisfying the given constraints.

(ii) Property ( $i$ ) also holds if $\alpha=k \in \mathbf{Z}_{0+}$ and either condition (C3) or condition (C4) of Theorem 16 holds.

Proof. Since rank $B(t)=n$ for all $t \in \mathbf{R}_{0+}$ and, since $m \geq n$, the right generalized inverse of $B(t), B_{\text {right }}^{-1}(t)=\left(B(t) B^{T}(t)\right)^{-1}$ exists for all $t \in \mathbf{R}_{0+}$ and then (89) is well-posed. One gets from Theorem 15, (84), after substituting (89) into (81)

$$
x(t) \geq\left(\frac{P_{N}+\rho_{N}}{(1-q)^{2 \alpha-k+1} t^{\alpha}} I-A_{0}(t)\right)^{-1} u_{0}(t) \geq 0
$$

$$
\forall t \in \mathbf{R}_{0+}
$$

since $u_{0}: \mathbf{R}_{0+} \rightarrow \mathbf{R}_{0+}^{m}$ and either condition (C1) or condition (C2) of Theorem 15 holds. Then, $x: \mathbf{R}_{0+} \rightarrow \mathbf{R}_{0+}^{n}$ and it is bounded since $u_{0}: \mathbf{R}_{0+} \rightarrow \mathbf{R}_{0+}^{m}$ is bounded. Note also that a similar upper-bounding expression for $x(t)$ can be got by rearranging signs in (81) resulting to be

$$
\begin{aligned}
& x(t) \leq\left(\frac{P_{N}+\rho_{N}}{(1-q)^{2 \alpha-k+1} t^{\alpha}} I-A_{0}(t)\right)^{-1} \\
& \times\left(\sum_{j=1}^{p} A_{j}(t) x\left(t-h_{j}\right)-\left(P_{N}+\rho_{N}\right)(1-q)^{k-\alpha}\right. \\
& \times\left[\widehat{S}_{N t}\left(\widehat{q}_{N} t\right)-\left(\frac{\gamma}{q^{k}}\right)^{N+1}\right. \\
& \times \frac{(k+1)\left(\|x(0)\|+\varepsilon_{N}\right)}{(1-\gamma)(1-q)^{k} t^{\alpha}} \\
& \left.\left.\times\left|\sum_{j=0}^{k}(-1)^{j}\left(\begin{array}{l}
k \\
j
\end{array}\right)\right| \widehat{e}\right]+B(t) u(t)\right) \geq 0 ;
\end{aligned}
$$

$\forall t \in \mathbf{R}_{0+}$

Then, the following properties hold. 
so that

$$
\begin{aligned}
0 \leq & \left(\frac{1}{(1-q)^{\alpha+1} t^{\alpha}} I-A_{0}(t)\right)^{-1} u_{0}(t) \leq x(t) \\
\leq & \left(\frac{1}{(1-q)^{\alpha+1} t^{\alpha}} I-A_{0}(t)\right)^{-1} \\
& \times\left(u_{0}(t)+\left(\frac{\gamma}{q^{k}}\right)^{N+1} \frac{(k+1)\left(\|x(0)\|+\varepsilon_{N}\right)}{(1-\gamma)(1-q)^{k} t^{\alpha}}\right. \\
& \left.\times\left|\sum_{j=0}^{k}(-1)^{j}\left(\begin{array}{c}
k \\
j
\end{array}\right)\right| \hat{e}\right) .
\end{aligned}
$$

Thus, if $u_{0}(t) \rightarrow 0$ as $t \rightarrow \infty$ then $x(t) \rightarrow 0$ as $t \rightarrow \infty$ since $\left(\gamma / q^{k}\right)^{N+1}(k+1)\left(\|x(0)\|+\varepsilon_{N}\right) /(1-\gamma)(1-q)^{k} t^{\alpha} \rightarrow 0$ as $t \rightarrow \infty$ and Property (i) has been proved. Property (ii) is similar to the solution satisfying

$$
x(t) \geq\left(\frac{1}{(1-q)^{\alpha+1} t^{\alpha}} I-A_{0}(t)\right)^{-1} u_{0}(t) \geq 0 ; \quad \forall t \in \mathbf{R}_{0+} .
$$

Note that $\left(\gamma / q^{k}\right)^{N+1}(k+1)\left(\|x(0)\|+\varepsilon_{N}\right) /(1-\gamma)(1-q)^{k} t^{\alpha}$ converges to zero as $N \rightarrow \infty$ and as $t \rightarrow \infty$. Thus, for any given $\varepsilon \in \mathbf{R}_{+}$and $N \in \mathbf{Z}_{+}$there is $t_{0}=t_{0}(N, \varepsilon) \in \mathbf{R}_{+}$such that

$$
\begin{aligned}
\left(\frac{\gamma}{q^{k}}\right)^{N+1} \frac{(k+1)\left(\|x(0)\|+\varepsilon_{N}\right)}{(1-\gamma)(1-q)^{k} t^{\alpha}}\left|\sum_{j=0}^{k}(-1)^{j}\left(\begin{array}{c}
k \\
j
\end{array}\right)\right| \leq \varepsilon ; \\
\forall t\left(\geq t_{0}\right) \in \mathbf{R}_{+} .
\end{aligned}
$$

As a result, if $u_{1}(t)$ in (90) is replaced with

$$
\begin{aligned}
& u_{1}(t)=u_{0}(t)-\sum_{j=1}^{p} A_{j}(t) x\left(t-h_{j}\right)+\left(P_{N}+\rho_{N}\right)(1-q)^{k-\alpha} \\
& \times \widehat{S}_{N t}\left(\widehat{q}_{N} t\right)+\left(\frac{\gamma}{q^{k}}\right)^{N+1} \\
& \times \frac{(k+1)\left(\|x(0)\|+\varepsilon_{N}\right)}{(1-\gamma)(1-q)^{k} t_{0}^{\alpha}} \\
&\left.\times\left|\sum_{j=0}^{k}(-1)^{j}\left(\begin{array}{c}
k \\
j
\end{array}\right)\right| \widehat{e}\right] .
\end{aligned}
$$

The following result is a consequence of Theorem 19 and Theorems 15-16 under a control law given by (89) and (96).

Corollary 20. Consider (55) under any set of bounded vector functions of initial conditions $\varphi_{j}:[-h, 0] \rightarrow \mathbf{R}_{0+}^{n}$, for all $j \in$ $\overline{k-1} \cup\{0\}$. Assume also that $B(t) \in \mathbf{R}_{+}^{n \times m}$, with $m \geq n$ and $\operatorname{rank} B(t)=n$, for all $t \in \mathbf{R}_{0+}$. Then, the following properties hold.

(i) If $\alpha \notin \mathbf{Z}_{0+}$ and the control law (89)-(90) is replaced with (89) and (96) then under either condition (C1) or condition (C2) of Theorem 15

$$
\begin{aligned}
& x(t) \geq\left(\frac{\left(P_{N}+\rho_{N}\right)}{(1-q)^{2 \alpha-k+1} t^{\alpha}} I-A_{0}(t)\right)^{-1} \\
& \times\left(u_{0}(t)+\left(\frac{\gamma}{q^{k}}\right)^{N+1} \frac{(k+1)\left(\|x(0)\|+\varepsilon_{N}\right)}{(1-\gamma)(1-q)^{k}}\right. \\
&\left.\times\left|\sum_{j=0}^{k}(-1)^{j}\left(\begin{array}{c}
k \\
j
\end{array}\right)\right|\left(\frac{1}{t_{0}^{\alpha}}-\frac{1}{t^{\alpha}}\right) \hat{e}\right) \geq 0 ; \\
& \quad \liminf _{t \rightarrow \infty}\left(x(t)-A_{0}^{-1}(t)\left(u_{0}(t)+\varepsilon \widehat{e}\right)\right) \geq 0
\end{aligned}
$$

for any given $\varepsilon \in \mathbf{R}_{+}$and $N \in \mathbf{Z}_{+}$and some $t_{0}=$ $t_{0}(N, \varepsilon) \in \mathbf{R}_{+}$. As a result, $\liminf _{t \rightarrow \infty}\left(x(t)-A_{0}^{-1} \varepsilon \widehat{e}\right) \geq$ 0 if $u_{0}(t) \rightarrow 0$ as $t \rightarrow \infty$.

(ii) If $\alpha=k \in \mathbf{Z}_{0+}$ and (90) is replaced with (96) for $k=\alpha$ then under either condition (C3) or condition (C4) of Theorem 16

$$
\begin{aligned}
& x(t) \geq\left(\frac{1}{(1-q)^{\alpha+1} t^{\alpha}} I-A_{0}(t)\right)^{-1} \\
& \times\left(u_{0}(t)+\left(\frac{\gamma}{q^{k}}\right)^{N+1} \frac{(k+1)\left(\|x(0)\|+\varepsilon_{N}\right)}{(1-\gamma)(1-q)^{k}}\right. \\
&\left.\times\left|\sum_{j=0}^{k}(-1)^{j}\left(\begin{array}{c}
k \\
j
\end{array}\right)\right|\left(\frac{1}{t_{0}^{\alpha}}-\frac{1}{t^{\alpha}}\right) \hat{e}\right) \geq 0 ; \\
& \forall t \in \mathbf{R}_{0+}
\end{aligned}
$$

and (98) holds.

Example 21. From Theorem 11, (57), one concludes that (55), under any set of nonnegative set of real vector functions of initial conditions on $[-h, 0]$ and any admissible nonnegative control vector function, has a limiting nonnegative $q$-solution of (Caputo) fractional order $\alpha \in \mathbf{Z}_{+}$satisfying

$$
\begin{aligned}
\liminf _{t \rightarrow \infty}(x(t) & -\left(\frac{1}{(1-q)^{\alpha+1} t^{\alpha}} I-A_{0}(t)\right)^{-1} \\
& \left.\times\left(\sum_{i=1}^{p} A_{i}(t) x\left(t-h_{i}\right)+B(t) u(t)\right)\right) \geq 0
\end{aligned}
$$

if $\exists\left(\left(1 /(1-q)^{\alpha+1} t^{\alpha}\right) I-A_{0}(t)\right)^{-1}$ for all $t \in \mathbf{R}_{0+}$ in order that the $q$-solution of (Caputo) fractional order $\alpha \in \mathbf{Z}_{+}$of (55) exist for $t \in \mathbf{R}_{0+}$ some of the two sets of constraints below holds: 
(1) One has $\liminf _{t \rightarrow \infty}\left(A_{0}(t)-\left(1 /(1-q)^{\alpha+1} t^{\alpha}\right) I\right)^{-1} \leq$ 0 , $\liminf _{t \rightarrow \infty} A_{i}(t) \geq 0$; for all $i \in \bar{p}$, $\liminf _{t \rightarrow \infty} B(t) \geq 0$.

The first above condition implies $\exists \lim _{t \rightarrow \infty}\left(-A_{0}^{-1}(t)\right)=-A_{0 \infty}^{-1} \geq 0$ so that $\left(-A_{0 \infty}\right)$ is a monomial matrix which implies that if $A_{0 \infty} \in M \mathbf{R}^{n \times n}$ then it is diagonal nonsingular with negative diagonal entries.

(2) One has $\exists \lim _{t \rightarrow \infty}\left(-A_{0}^{-1}(t)\right)=-A_{0 \infty}^{-1}$, $-A_{0 \infty}^{-1}\left(\liminf _{t \rightarrow \infty} A_{i}(t)\right) \geq 0$; for all $i \in \bar{p}$, $-A_{0 \infty}^{-1}\left(\liminf _{t \rightarrow \infty} B(t)\right) \geq 0$.

Note that it has been used that for $\alpha>0, \widehat{S}_{N}\left(\widehat{q}_{N} t\right) \rightarrow 0$, $\widetilde{S}_{N}\left(\widehat{q}_{N} t\right) \rightarrow 0$ as $t \rightarrow \infty$, for all $N \in \mathbf{Z}_{0+}$ from (47)-(48).

If $\alpha=0$ then the existence of $A_{0 \infty}^{-1}$ is not required and the first constraint of each of the above sets is changed to the existence of $\liminf _{t \rightarrow \infty}\left(A_{0}(t)-(1-q)^{-1} I\right)^{-1} \leq$ 0 , that is, $\limsup _{t \rightarrow \infty} A_{0}(t) \leq(1-q)^{-1} I$ provided that $\lim \sup _{t \rightarrow \infty}\left\|A_{0}(t)\right\|_{2}<(1-q)^{-1}$ to guarantee the nonsingularity of $\left(A_{0}(t)-(1-q)^{-1} I\right)$.

If $\alpha \notin \mathbf{Z}_{0+}$ and $k=[\alpha]+1$ then the above constraints have to be amended via (56) with the replacement $((1 /(1-$ $\left.\left.q)^{\alpha+1} t^{\alpha}\right) I\right) \rightarrow\left(\left(P^{*} /(1-q)^{2 \alpha-\mathrm{k}+1} t^{\alpha}\right) I\right)$.

On the other hand, note that the various above results and properties given in the above remarks within this section lead directly to sufficiency-type conditions for the nonnegativity of the quantum fractional solutions and their limit values under the proposed control laws.

Remark 22. If the q-Caputo fractional differential system (61) is considered instead of (55), then Theorem 19 and Corollary 20 hold under modified equations (90), (92), (93), (95), and (96) being subject to replacements (87) and $\widehat{S}_{N t}\left(\widetilde{q}_{N} t\right) \rightarrow\left(\widehat{S}_{N(t-\zeta)}\left(\widetilde{q}_{N}(t-\zeta)\right)-\widehat{S}_{N t}\left(\widetilde{q}_{N} t\right)\right)$ for any given $\zeta \in \mathbf{R}_{+}$and for all $t(\geq \zeta) \in \mathbf{R}_{+}$(see Remark 17). On the other hand, modified equations (97) and (99) remain valid under the above replacements together with the additional replacements $1 / t_{0}^{\alpha} \rightarrow\left(1 / t_{0}^{\alpha}-1 /\left(t_{0}-\zeta\right)^{\alpha}\right)$ and $1 / t^{\alpha} \rightarrow$ $\left(1 / t^{\alpha}-1 /(t-\zeta)^{\alpha}\right)$. A variant of the disposal can be made with the replacement of $\zeta$ with a function $\zeta: \mathbf{R}_{0+} \rightarrow \mathbf{R}_{+}$with $\zeta(t) \leq t$, for all $t \in \mathbf{R}_{0+}$.

Remark 23. In the delay-free case and in the case that the delays are time-varying, the given formalism is extendable under minor modifications. For instance, assume that the delays satisfy the constraints $t-h_{i}(t)=q^{-c_{i}(t) t}$, for all $i \in \bar{p}$, for all $t \in \mathbf{R}_{0+}$ for a set of positive real constants satisfying $c_{i}(t)<c_{i+1}(t)$, for all $i \in \overline{p-1}$, for all $t \in \mathbf{R}_{0+}$. Thus, an appropriate solution of (61) in discretized form can be organized on intervals $\left[t_{i}-\zeta_{i}\left(t_{i}\right), t_{i}\right]$, for all $i \in \mathbf{Z}_{+}$according to the subsequent algorithm.

Step 1 (initial point). Select an initial point $t_{1} \in \mathbf{R}_{0+}$.

Step 2 (sequence of points to construct the discrete solution). Fix a strictly increasing string of positive real numbers $\left(t_{1}, t_{2}, t_{3}, \ldots\right)$ such that $t_{i+1}=q^{-m_{i}\left(t_{i}\right)} t_{i}$ for all $i \in \mathbf{Z}_{+}$and some given sequence $\left\{m_{i}\left(t_{i}\right)\right\}$ of positive integers.

Step 3 (sequence of interval lengths to construct the discrete solution recursively). Fix a string of interval (nonzero) measures $\left(\zeta_{1}\left(t_{1}\right), \zeta_{2}\left(t_{2}\right), \zeta_{3}\left(t_{3}\right), \ldots\right)$, which are the measures of the computation intervals $\left[t_{i}-\zeta_{i}\left(t_{i}\right), t_{i}\right]$, for all $i \in \mathbf{Z}_{+}$in (61), such that $\zeta_{i+1}\left(t_{i+1}\right)=q^{-j_{i}\left(t_{i+1}\right)} t_{i}$, for all $i \in \mathbf{Z}_{+}$for some sequence $\left\{j_{i}\left(t_{i+1}\right)\right\}$, of nonnegative integers, with $j_{i}\left(t_{i+1}\right) \leq$ $m_{i}\left(t_{i}\right)$, satisfying $1 \leq q^{-m_{i}\left(t_{i}\right)}-q^{-j_{i}\left(t_{i+1}\right)}$, for all $i \in \mathbf{Z}_{+}$so as to guarantee the algorithm running constraints:

$$
t_{i} \leq\left(q^{-m_{i}\left(t_{i}\right)}-q^{-j_{i}\left(t_{i+1}\right)}\right) t_{i}<t_{i+1}=q^{-m_{i}\left(t_{i}\right)} t_{i} ; \quad \forall i \in \mathbf{Z}_{+} .
$$

\section{Conflict of Interests}

The author declares that there is no conflict of interests regarding the publication of this paper.

\section{Acknowledgments}

The author is very grateful to the Spanish Government for its support through Grant DPI2012-30651 and to the Basque Government for its support through Grants IT378-10 and SAIOTEK S-PE13UN039. He is also grateful to the University of Basque Country for its support through Grant UFI 2011/07 and to the referees for their useful comments on the first version of the paper.

\section{References}

[1] A. A. Kilbas, H. M. Srivastava, and J. J. Trujillo, Theory and Applications of Fractional Differential Equations, vol. 204 of North-Holland Mathematics Studies, Elsevier, Amsterdam, The Netherlands, 2006.

[2] G. T. Wang, S. Y. Liu, D. Baleanu, and L. H. Zhang, "Existence results for nonlinear fractional differential equations involving different Riemann-Liouville fractional derivatives," Advances in Difference Equations, vol. 2013, article 280, 2013.

[3] Yu. F. Luchko and H. M. Srivastava, "The exact solution of certain differential equations of fractional order by using operational calculus," Computers \& Mathematics with Applications, vol. 29, no. 8, pp. 73-85, 1995.

[4] M. A. Darwish, "On quadratic integral equation of fractional orders," Journal of Mathematical Analysis and Applications, vol. 311, no. 1, pp. 112-119, 2005.

[5] M. A. Darwish, "Nondecreasing solutions of a fractional quadratic integral equation of Urysohn-Volterra type," Dynamic Systems and Applications, vol. 20, no. 4, pp. 423-437, 2011.

[6] D. Baleanu, P. Agarwal, and S.D. Purohit, "Certain fractional integral formulas involving the product of generalized bessel functions," The Scientific World Journal, vol. 2013, Article ID 567132, 9 pages, 2013.

[7] A. Babakhani and V. Daftardar-Gejji, "On calculus of local fractional derivatives," Journal of Mathematical Analysis and Applications, vol. 270, no. 1, pp. 66-79, 2002. 
[8] A. Ashyralyev, "A note on fractional derivatives and fractional powers of operators," Journal of Mathematical Analysis and Applications, vol. 357, no. 1, pp. 232-236, 2009.

[9] B. Baeumer, M. M. Meerschaert, and J. Mortensen, "Space-time fractional derivative operators," Proceedings of the American Mathematical Society, vol. 133, no. 8, pp. 2273-2282, 2005.

[10] H. M. Srivastava and P. Agarwal, "Certain fractional integral operators and the generalized incomplete hypergeometric functions," Applications and Applied Mathematics, vol. 8, no. 2, pp. 480-487, 2013.

[11] P. Agarwal and M. Chand, "Graphical analysis of Kampé de Fériet's series with Implementation of MATLAB," International Journal of Computer Applications, vol. 59, no. 4, pp. 1-10, 2012.

[12] A. M. A. El-Sayed and M. Gaber, "On the finite Caputo and finite Riesz derivatives," Electronic Journal of Theoretic Physics, vol. 3 , no. 12, pp. 81-95, 2006.

[13] R. Almeida, A. B. Malinowska, and D. F. M. Torres, "A fractional calculus of variations for multiple integrals with application to vibrating string," Journal of Mathematical Physics, vol. 51, no. 3, Article ID 033503, 12 pages, 2010.

[14] I. Schäfer and S. Kempfle, "Impulse responses of fractional damped systems," Nonlinear Dynamics, vol. 38, no. 1-4, pp. 6168, 2004.

[15] F. J. Molz, III, G. J. Fix, III, and S. Lu, "A physical interpretation for the fractional derivative in Levy diffusion," Applied Mathematics Letters, vol. 15, no. 7, pp. 907-911, 2002.

[16] M. Ilic, I. W. Turner, F. Liu, and V. Anh, "Analytical and numerical solutions of a one-dimensional fractional-in-space diffusion equation in a composite medium," Applied Mathematics and Computation, vol. 216, no. 8, pp. 2248-2262, 2010.

[17] M. D. Ortigueira, "On the initial conditions in continuous-time fractional linear systems," Signal Processing, vol. 83, no. 11, pp. 2301-2309, 2003.

[18] J. Lancis, T. Szoplik, E. Tajahuerce, V. Climent, and M. Fernández-Alonso, "Fractional derivative Fourier plane filter for phase-change visualization," Applied Optics, vol. 36, no. 29, pp. 7461-7464, 1997.

[19] E. Scalas, R. Gorenflo, and F. Mainardi, "Fractional calculus and continuous-time finance," Physica A, vol. 284, no. 1-4, pp. 376$384,2000$.

[20] S. Das, Functional Fractional Calculus for System Identification and Controls, Springer, Berlin, Germany, 2008.

[21] J. A. Tenreiro MacHado, M. F. Silva, R. S. Barbosa et al., "Some applications of fractional calculus in engineering," Mathematical Problems in Engineering, vol. 2010, Article ID 639801, 34 pages, 2010.

[22] J. Sabatier, O. P. Agrawal, and J. A. Tenreiro Machado, Eds., Advances in Fractional Calculus: Theoretical Developments and Applications in Physics and Engineering, Springer, Dordrecht, The Netherlands, 2007.

[23] P. Agarwal, "New unified integrals involving Sirivastava polynomials and H-function," Journal of Fractional Calculus and Applications, vol. 3, no. 3, pp. 1-7, 2012.

[24] H. M. Sirivastava and P. Agarwal, "Certain fractional integral operators and the generalized incomplete hypergeometric functions," Applications and Applied Mathematics, vol. 8, no. 2, pp. 333-345, 2013.

[25] M. De la Sen, "Stability of impulsive time-varying systems and compactness of the operators mapping the input space into the state and output spaces," Journal of Mathematical Analysis and Applications, vol. 321, no. 2, pp. 621-650, 2006.
[26] K. Ratchagit, "New delay-dependent conditions for the robust stability of linear polytopic discrete-time systems," Journal of Computational Analysis and Applications, vol. 13, no. 3, pp. 463469, 2011.

[27] K. Ratchagit, "Asymptotic stability of linear continuous timevarying systems with state delays in Hilbert spaces," Journal of Computational Analysis and Applications, vol. 13, no. 3, pp. 554564, 2011.

[28] M. De La Sen, "On the reachability and controllability of positive linear time-invariant dynamic systems with internal and external incommensurate point delays," The Rocky Mountain Journal of Mathematics, vol. 40, no. 1, pp. 177-207, 2010.

[29] M. De la Sen, "A method for general design of positive real functions," IEEE Transactions on Circuits and Systems I: Fundamental Theory and Applications, vol. 45, no. 7, pp. 764769, 1998.

[30] M. De la Sen, "Preserving positive realness through discretization," Positivity, vol. 6, no. 1, pp. 31-45, 2002.

[31] M. De la Sen, "On positivity of singular regular linear timedelay time-invariant systems subject to multiple internal and external incommensurate point delays," Applied Mathematics and Computation, vol. 190, no. 1, pp. 382-401, 2007.

[32] M. De la Sen, "Positivity and stability of the solutions of Caputo fractional linear time-invariant systems of any order with internal point delays," Abstract and Applied Analysis, vol. 2011, Article ID 161246, 25 pages, 2011.

[33] M. De la Sen, "On Chebyshev's systems and non-uniform sampling related to Caputo fractional dynamic time-invariant systems," Discrete Dynamics in Nature and Society, vol. 2010, Article ID 846590, 24 pages, 2010.

[34] M. De la Sen, "About robust stability of Caputo linear fractional dynamic systems with time delays through fixed point theory," Fixed Point Theory and Applications, vol. 2011, Article ID 867932, 19 pages, 2011.

[35] Y. Zhao, H. Chen, and Q. Zhang, "Existence and multiplicity of positive solutions for nonhomogeneous boundary value problems with fractional $q$-derivatives," Boundary Value Problems, vol. 2013, article 103, 16 pages, 2013.

[36] P. M. Rajković, S. D. Marinković, and M. S. Stanković, "Fractional integrals and derivatives in q-calculus," Applicable Analysis and Discrete Mathematics, vol. 1, no. 1, pp. 311-323, 2007.

[37] A. Aral, V. Gupta, and R. P. Agarwal, Applications of q-Calculus in Operator Theory, Springer, New York, NY, USA, 2013.

[38] F. Jarad, T. Abdeljawad, and D. Baleanu, "Stability of $q$-fractional non-autonomous systems," Nonlinear Analysis: Real World Applications, vol. 14, no. 1, pp. 780-784, 2013.

[39] V. Kac and P. Cheung, Quantum Calculus, Universitext, Springer, New York, NY, USA, 2002.

[40] F. M. Atici and P. W. Eloe, "Fractional q-calculus on a time scale," Journal of Nonlinear Mathematical Physics, vol. 14, no. 3, pp. 333-344, 2007.

[41] T. Abdeljawad and D. Baleanu, "Caputo $q$-fractional initial value problems and a $q$-analogue Mittag-Leffler function," Communications in Nonlinear Science and Numerical Simulation, vol. 16, no. 12, pp. 4682-4688, 2011.

[42] G.-C. Wu and D. Baleanu, "New applications of the variational iteration method-from differential equations to $q$-fractional difference equations," Advances in Difference Equations, vol. 2013, article 21, 16 pages, 2013.

[43] M. H. Annaby and Z. S. Mansour, q-fractional Calculus and Equations, vol. 2056 of Lecture Notes in Mathematics Series, Springer, Heidelberg, Germany, 2012. 
[44] X. J. Yang and D. Baleanu, "Fractal heat conduction problem solved by local fractional variation iteration method," Thermal Science, vol. 17, no. 2, pp. 625-628, 2013.

[45] A. M. Yang, Z. S. Chen, H. M. Srivistava, and X. J. Yang, "Application of the local fractional series expansion method and the variational iteration method to the Helmholtz equation involving local fractional derivative operators," Abstract and Applied Analysis, vol. 2013, Article ID 259125, 6 pages, 2013. 


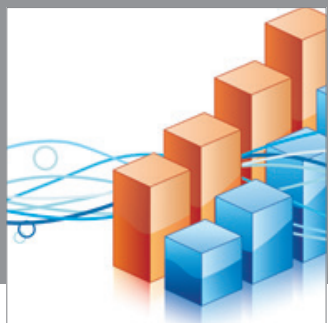

Advances in

Operations Research

mansans

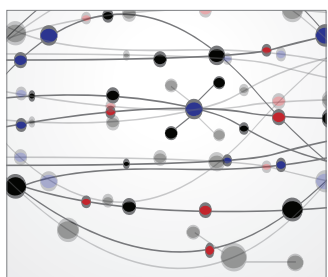

The Scientific World Journal
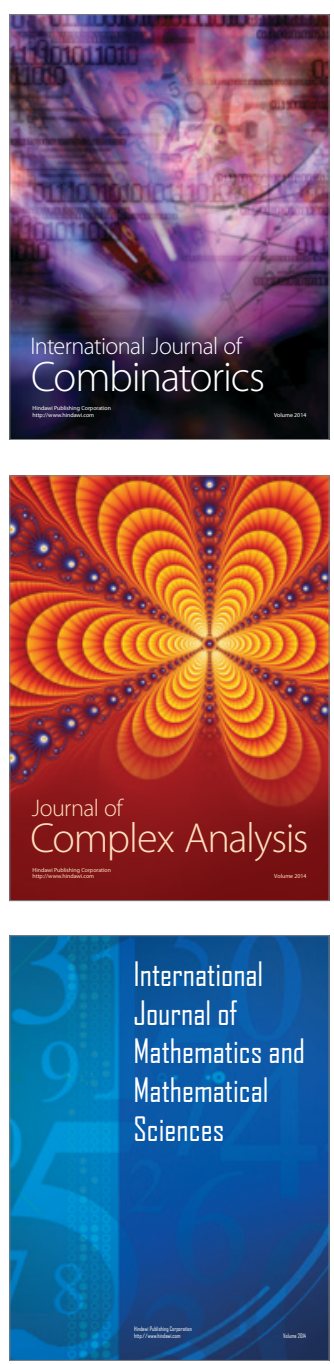
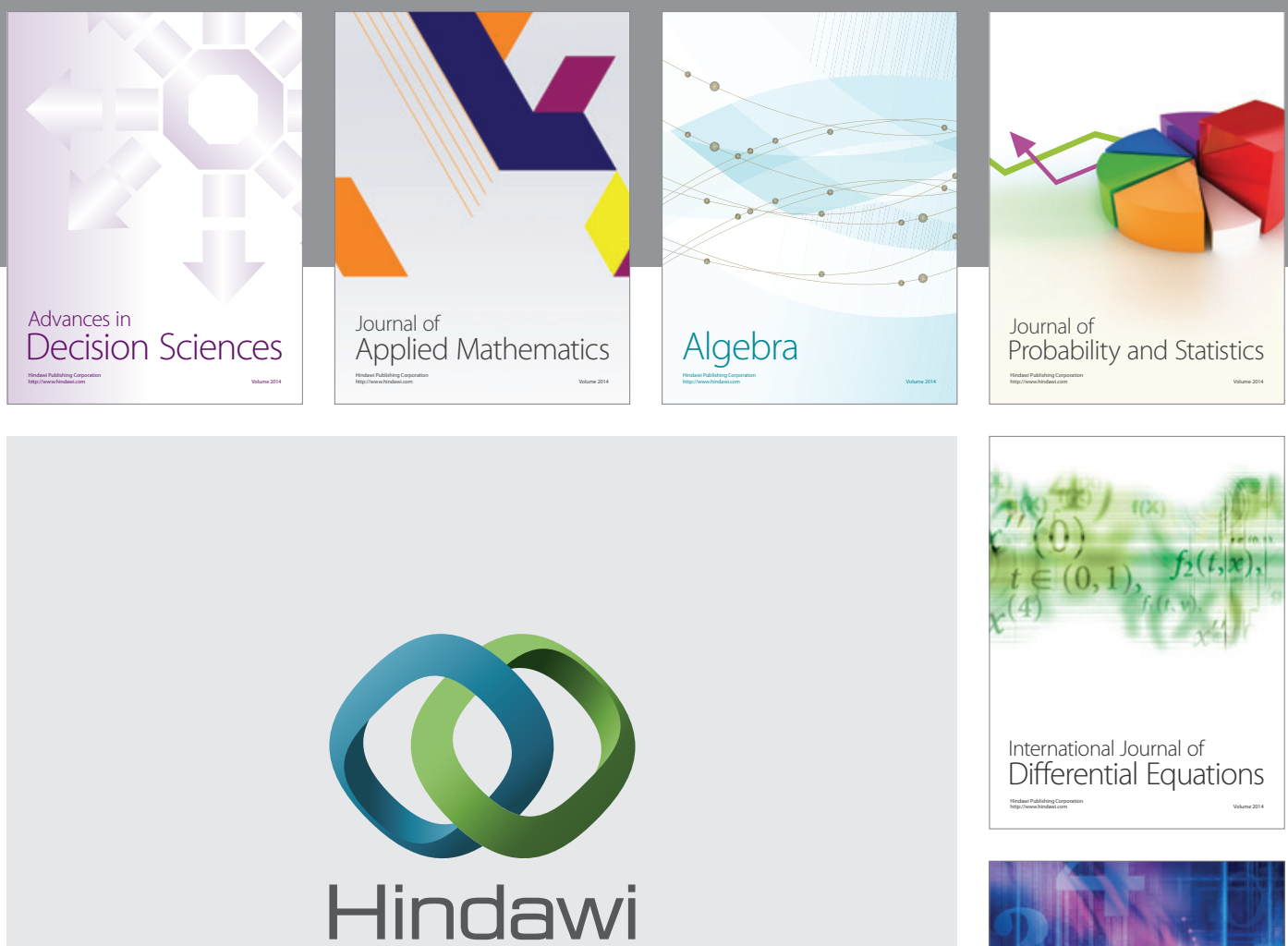

Submit your manuscripts at http://www.hindawi.com
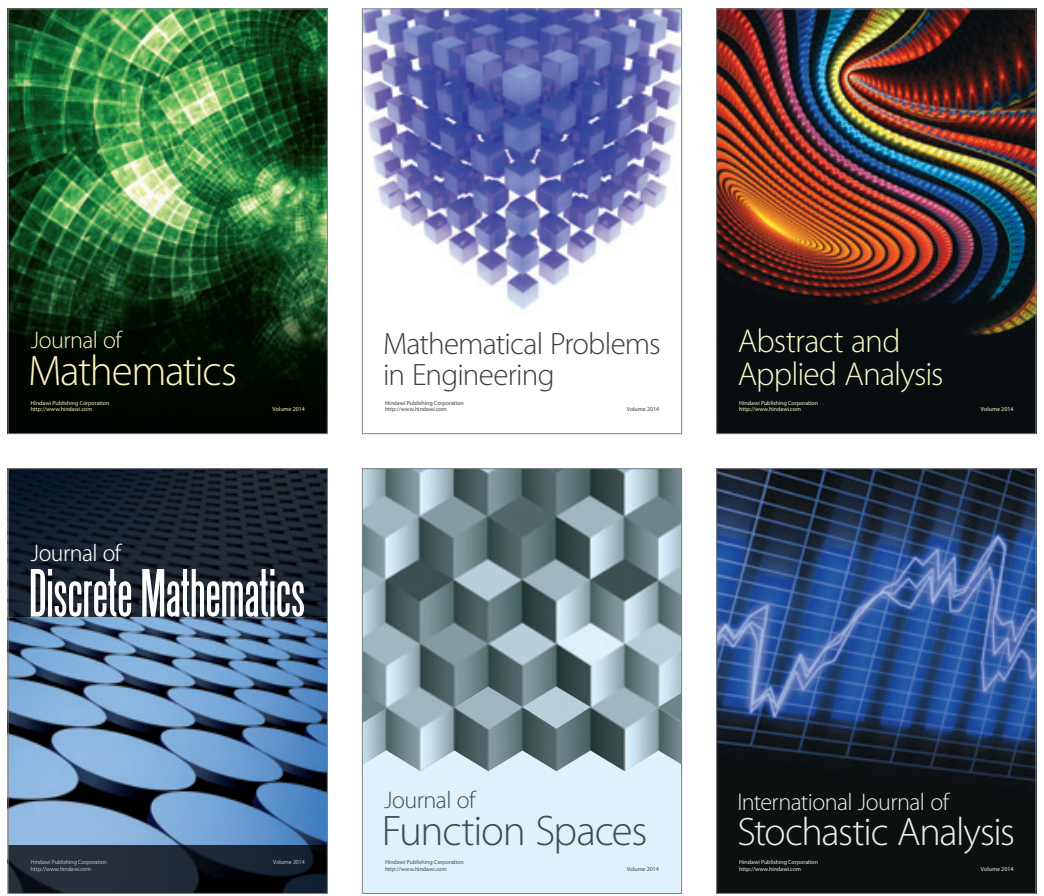

Journal of

Function Spaces

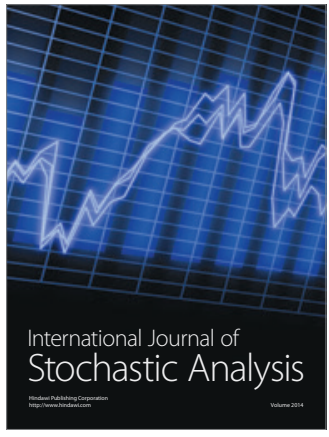

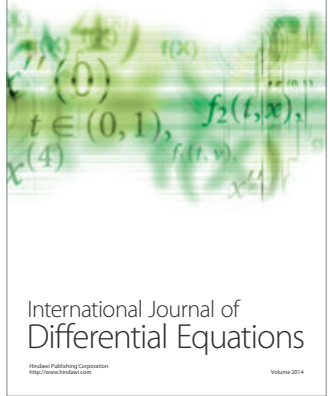
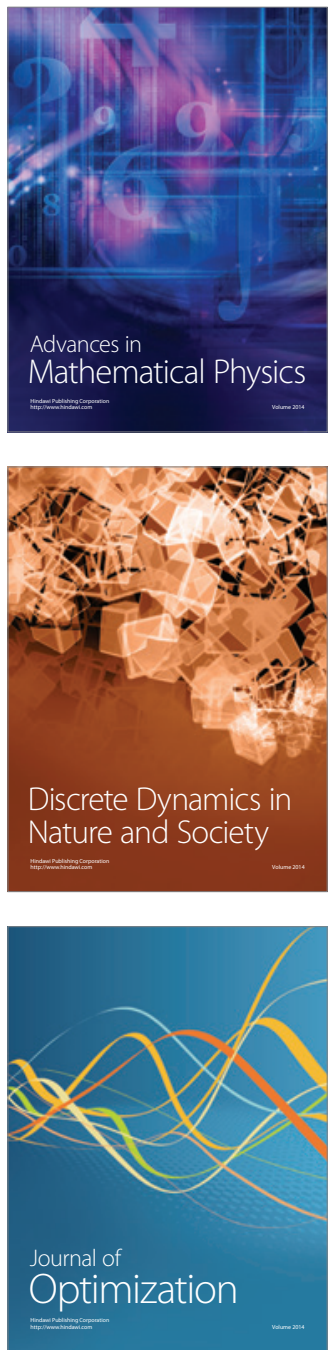